\title{
THE CLOSURE OF TWO-SIDED MULTIPLICATIONS ON C*-ALGEBRAS AND PHANTOM LINE BUNDLES
}

\author{
ILJA GOGIĆ ${ }^{1,2}$ AND RICHARD M. TIMONEY ${ }^{2}$
}

\begin{abstract}
For a $C^{*}$-algebra $A$ we consider the problem of when the set $\mathrm{TM}_{0}(A)$ of all two-sided multiplications $x \mapsto a x b(a, b \in A)$ on $A$ is norm closed, as a subset of $\mathcal{B}(A)$. We first show that $\operatorname{TM}_{0}(A)$ is norm closed for all prime $C^{*}$-algebras $A$. On the other hand, if $A \cong \Gamma_{0}(\mathcal{E})$ is an $n$-homogeneous $C^{*}$-algebra, where $\mathcal{E}$ is the canonical $\mathbb{M}_{n}$-bundle over the primitive spectrum $X$ of $A$, we show that $\mathrm{TM}_{0}(A)$ fails to be norm closed if and only if there exists a $\sigma$-compact open subset $U$ of $X$ and a phantom complex line subbundle $\mathcal{L}$ of $\mathcal{E}$ over $U$ (i.e. $\mathcal{L}$ is not globally trivial, but is trivial on all compact subsets of $U$ ). This phenomenon occurs whenever $n \geq 2$ and $X$ is a CW-complex (or a topological manifold) of dimension $3 \leq d<\infty$.
\end{abstract}

\section{INTRODUCTION}

Let $A$ be a $C^{*}$-algebra and let $\operatorname{IB}(A)$ (resp. $\left.\operatorname{ICB}(A)\right)$ denote the set of all bounded (resp. completely bounded) maps $\phi: A \rightarrow A$ that preserve (closed two-sided) ideals of $A$ (i.e. $\phi(I) \subseteq I$ for all ideals $I$ of $A$ ). The most prominent class of maps $\phi \in \operatorname{ICB}(A) \subset \operatorname{IB}(A)$ are elementary operators, i.e. those that can be expressed as finite sums of two-sided multiplications $M_{a, b}: x \mapsto a x b$, where $a$ and $b$ are elements of the multiplier algebra $M(A)$.

Elementary operators play an important role in modern quantum information and quantum computation theory. In particular, maps $\phi: \mathbb{M}_{n} \rightarrow \mathbb{M}_{n}\left(\mathbb{M}_{n}\right.$ are $n \times n$ matrices over $\mathbb{C}$ ) of the form $\phi=\sum_{i=1}^{\ell} M_{a_{i}^{*}, a_{i}}\left(a_{i} \in \mathbb{M}_{n}\right.$ such that $\left.\sum_{i=1}^{\ell} a_{i}^{*} a_{i}=1\right)$ represent the (trace-duals of) quantum channels, which are mathematical models of the evolution of an 'open' quantum system (see e.g. 21]). Elementary operators also provide ways to study the structure of $C^{*}$-algebras (see [2]).

Let $\mathcal{E} \ell(A), \operatorname{TM}(A)$ and $\mathrm{TM}_{0}(A)$ denote, respectively, the sets of all elementary operators on $A$, two-sided multiplications on $A$ and two-sided multiplications on $A$ with coefficients in $A$ (i.e. $\operatorname{TM}_{0}(A)=\left\{M_{a, b}: a, b \in A\right\}$ ).

The elementary operators are always dense in $\operatorname{IB}(A)$ in the topology of pointwise convergence (by [23, Corollary 2.3]). However, more subtle considerations enter in when one asks if $\phi \in \operatorname{ICB}(A)$ can be approximated pointwise by elementary operators of cb-norm at most $\|\phi\|_{c b}$ (24] shows that nuclearity of $A$ suffices; see also [26]).

Date: Version April 5, 2018.

2010 Mathematics Subject Classification. Primary 46L05, 46M20; Secondary 46L07.

Key words and phrases. $C^{*}$-algebra, homogeneous, two-sided multiplication, elementary operator, complex line bundle.

The work of the first author was supported by the Irish Research Council under grant GOIPD $/ 2014 / 7$.

The work of the second author was supported by the Science Foundation Ireland under grant 11/RFP/MTH3187. 
It is an interesting problem to describe those operators $\phi \in \operatorname{IB}(A)$ (resp. $\phi \in$ $\operatorname{ICB}(A))$ that can be approximated in operator norm (resp. cb-norm) by elementary operators. Earlier works, which we cite below, revealed that this is an intricate question in general, and can involve many and varied properties of $A$ and $\phi$. In this paper, we show that the apparently much simpler problems of describing the norm closures of $\operatorname{TM}(A)$ and $\mathrm{TM}_{0}(A)$ can have complicated answers even for rather well-behaved $C^{*}$-algebras.

In some cases $\mathcal{E} \ell(A)=\operatorname{IB}(A)$ (which implies $\mathcal{E} \ell(A)=\operatorname{ICB}(A)$ ); or $\mathcal{E} \ell(A)$ is norm dense in $\operatorname{IB}(A)$; or $\mathcal{E} \ell(A) \subset \operatorname{ICB}(A)$ is dense in cb-norm. The conditions just mentioned are in fact all equivalent for separable $C^{*}$-algebras $A$. More precisely, Magajna 25] shows that for separable $C^{*}$-algebras $A$, the property that $\mathcal{E} \ell(A)$ is norm (resp. cb-norm) dense in $\operatorname{IB}(A)$ (resp. $\operatorname{ICB}(A)$ ) characterizes finite direct sums of homogeneous $C^{*}$-algebras with the finite type property. Moreover, in this situation we already have the equality $\operatorname{ICB}(A)=\operatorname{IB}(A)=\mathcal{E} \ell(A)$.

It can happen that $\mathcal{E} \ell(A)$ is already norm closed (or cb-norm closed). In 13, 14, the first author showed that for a unital separable $C^{*}$-algebra $A$, if $\mathcal{E} \ell(A)$ is norm (or cb-norm) closed then $A$ is necessarily subhomogeneous, the homogeneous subquotients of $A$ must have the finite type property and established further necessary conditions on $A$. In 14, 15, he gave some partial converse results.

There is a considerable literature on derivations and inner derivations of $C^{*}$ algebras. Inner derivations $d_{a}$ on a $C^{*}$-algebra $A$, (i.e. those of the form $d_{a}(x)=$ $a x-x a$ with $a \in M(A))$ are important examples of elementary operators. In 35, Corollary 4.6] Somerset shows that if $A$ is unital, $\left\{d_{a}: a \in A\right\}$ is norm closed if and only if $\operatorname{Orc}(A)<\infty$, where $\operatorname{Orc}(A)$ is a constant defined in terms of a certain graph structure on $\operatorname{Prim}(\mathrm{A})$ (the primitive spectrum of $A$ ). If $\operatorname{Orc}(A)=\infty$, the structure of outer derivations that are norm limits of inner derivations remains undescribed. In addition, if $A$ is unital and separable, then by [19, Theorem 5.3] and [35. Corollary 4.6] $\operatorname{Orc}(A)<\infty$ if and only if the set $\left\{M_{u, u^{*}}: u \in A, u\right.$ unitary $\}$ of inner automorphisms is norm closed.

In [12, 15 the first author considered the problem of which derivations on unital $C^{*}$-algebras $A$ can be cb-norm approximated by elementary operators. By [15, Theorem 1.5] every such a derivation is necessarily inner in a case when every Glimm ideal of $A$ is prime. When this fails, it is possible to produce examples which have outer derivations that are simultaneously elementary operators ([12, Example 6.1]).

While considering derivations $d$ that are elementary operators and/or norm limits of inner derivations, we realized that they are sometimes expressible in the form $d=M_{a, b}-M_{b, a}$ even though they are not inner. We have not been able to decide when all such $d$ are of this form, but this led us to the seemingly simpler question of considering the closures of $\operatorname{TM}(A)$ and $\operatorname{TM}_{0}(A)$. In this paper, we see that nontrivial considerations enter into these questions about two-sided multiplications. Of course the left multiplications $\left\{M_{a, 1}: a \in M(A)\right\}$ are already norm closed, as are the right multiplications. $\operatorname{So} \operatorname{TM}(A)$ is a small subclass of $\mathcal{E} \ell(A)$, and seems to be the basic case to study.

This paper is organized as follows. We begin in $\$ 2$ with some generalities and an explanation that the set of elementary operators of length at most $\ell$ has the same completion in the operator and cb-norms (for each $\ell \geq 1$ ). 
In 93 we show that for a prime $C^{*}$-algebra $A$, we always have $\operatorname{TM}(A)$ and $\mathrm{TM}_{0}(A)$ both norm closed.

In 4 , we recall the description of $\left(n\right.$-)homogeneous $C^{*}$-algebras $A$ as sections $\Gamma_{0}(\mathcal{E})$ of $\mathbb{M}_{n}$-bundles $\mathcal{E}$ over $X=\operatorname{Prim}(A)$ and some general results about $\operatorname{IB}(A)$, $\operatorname{ICB}(A)$ and $\mathcal{E} \ell(A)$ for such $A$.

In 95 for homogeneous $C^{*}$-algebras $A=\Gamma_{0}(\mathcal{E})$, we consider subclasses $\operatorname{IB}_{1}(A)$ and $\mathrm{IB}_{0,1}(A)$ of $\operatorname{IB}(A)$ that seem (respectively) to be the most obvious choices for the norm closure of $\operatorname{TM}(A)$ and of $\operatorname{TM}_{0}(A)$, extrapolating from fibrewise restrictions on $\phi \in \operatorname{TM}(A)$. For each $\phi \in \operatorname{IB}_{1}(A)$ we associate a complex line subbundle $\mathcal{L}_{\phi}$ of the restriction $\left.\mathcal{E}\right|_{U}$ to an open subset $U \subseteq X=\operatorname{Prim}(A)$, where $U$ is determined by $\phi$ as the cozero set of $\phi$ ( $U$ identifies the fibres of $\mathcal{E}$ on which $\phi$ acts by a nonzero operator). For separable $A$, the main result of this section is Theorem 5.15 where we characterize the condition $\phi \in \operatorname{TM}(A)$ in terms of triviality of the bundle $\mathcal{L}_{\phi}$. We close $\$ 5$ with Remark 5.21 comparing our bundle considerations to slightly similar results in the literature for innerness of $C(X)$-linear automorphisms when $X$ is compact, or for some more general unital $A$.

Our final 6 is the main section of this paper. For homogeneous $C^{*}$-algebras $A=\Gamma_{0}(\mathcal{E})$, we characterize operators $\phi$ in the norm closure of $\operatorname{TM}_{0}(A)$ as those operators in $\operatorname{IB}_{0,1}(A)$ for which the associated complex line bundle $\mathcal{L}_{\phi}$ is trivial on each compact subset of $U$, where $U$ is as above (Theorem 6.9). As a consequence, we obtain that $\operatorname{TM}_{0}(A)$ fails to be norm closed if and only if there exists a $\sigma$-compact open subset $U$ of $X$ and a phantom complex line subbundle $\mathcal{L}$ of $\left.\mathcal{E}\right|_{U}$ (i.e. $\mathcal{L}$ is not globally trivial, but is trivial on each compact subset of $U$ ). Using this and some algebraic topological ideas, we show that $\operatorname{TM}(A)$ and $\operatorname{TM}_{0}(A)$ both fail to be norm closed whenever $A$ is $n$-homogeneous with $n \geq 2$ and $X$ contains an open subset homeomorphic to $\mathbb{R}^{d}$ for some $d \geq 3$ (Theorem 6.18).

\section{Preliminaries}

Throughout this paper $A$ will denote a $C^{*}$-algebra. By an ideal of $A$ we always mean a closed two-sided ideal. As usual, by $Z(A)$ we denote the centre of $A$, by $M(A)$ the multiplier algebra of $A$, and by $\operatorname{Prim}(A)$ the primitive spectrum of $A$ (i.e. the set of kernels of all irreducible representations of $A$ equipped with the Jacobson topology).

Every $\phi \in \operatorname{IB}(A)$ is linear over $Z(M(A))$ and, for any ideal $I$ of $A, \phi$ induces a map

$$
\phi_{I}: A / I \rightarrow A / I, \quad \text { which sends } a+I \text { to } \phi(a)+I .
$$

It is easy to see that the norm (resp. cb-norm) of an operator $\phi \in \operatorname{IB}(A)$ (resp. $\phi \in \operatorname{ICB}(A))$ can be computed via the formulae

$$
\begin{aligned}
\|\phi\| & =\sup \left\{\left\|\phi_{P}\right\|: P \in \operatorname{Prim}(A)\right\} \quad \text { resp. } \\
\|\phi\|_{c b} & =\sup \left\{\left\|\phi_{P}\right\|_{c b}: P \in \operatorname{Prim}(A)\right\} .
\end{aligned}
$$

The length of a non-zero elementary operator $\phi \in \mathcal{E} \ell(A)$ is the smallest positive integer $\ell=\ell(\phi)$ such that $\phi=\sum_{i=1}^{\ell} M_{a_{i}, b_{i}}$ for some $a_{i}, b_{i} \in M(A)$. We also define $\ell(0)=0$. We write $\mathcal{E} \ell_{\ell}(A)$ for the elementary operators of length at most $\ell$. Thus $\mathcal{E} \ell_{1}(A)=\operatorname{TM}(A)$. 
We will also consider the following subsets of $\operatorname{TM}(A)$ :

$$
\begin{aligned}
\operatorname{TM}_{c p}(A) & =\left\{M_{a, a^{*}}: a \in M(A)\right\}, \\
\operatorname{Inn}_{\operatorname{Aut}}(A) & =\left\{M_{a, a^{-1}}: a \in M(A), a \text { invertible }\right\}, \text { and } \\
\operatorname{Inn} A u t(A) & =\left\{M_{u, u^{*}}: u \in M(A), u \text { unitary }\right\}
\end{aligned}
$$

(where cp and alg signify, respectively, completely positive and algebraic). Note that $\operatorname{InnAut}(A)=\mathrm{TM}_{c p}(A) \cap \operatorname{InnAutalg}(A)$.

It is well known that elementary operators are completely bounded with the following estimate for their cb-norm:

$$
\left\|\sum_{i} M_{a_{i}, b_{i}}\right\|_{c b} \leq\left\|\sum_{i} a_{i} \otimes b_{i}\right\|_{h},
$$

where $\|\cdot\|_{h}$ is the Haagerup tensor norm on the algebraic tensor product $M(A) \otimes$ $M(A)$, i.e.

$$
\|u\|_{h}=\inf \left\{\left\|\sum_{i} a_{i} a_{i}^{*}\right\|^{\frac{1}{2}}\left\|\sum_{i} b_{i}^{*} b_{i}\right\|^{\frac{1}{2}}: u=\sum_{i} a_{i} \otimes b_{i}\right\} .
$$

By inequality (2.4) the mapping

$$
\left(M(A) \otimes M(A),\|\cdot\|_{h}\right) \rightarrow\left(\mathcal{E} \ell(A),\|\cdot\|_{c b}\right) \quad \text { given by } \quad \sum_{i} a_{i} \otimes b_{i} \mapsto \sum_{i} M_{a_{i}, b_{i}} .
$$

defines a well-defined contraction. Its continuous extension to the completed Haagerup tensor product $M(A) \otimes_{h} M(A)$ is known as a canonical contraction from $M(A) \otimes_{h}$ $M(A)$ to $\operatorname{ICB}(A)$ and is denoted by $\Theta_{A}$.

We have the following result (see [2, Proposition 5.4.11]):

Theorem 2.1 (Mathieu). $\Theta_{A}$ is isometric if and only if $A$ is a prime $C^{*}$-algebra.

The next result is a combination of [36. Corollary 3.8], (2.2) and the facts that for $\phi=\sum_{i=1}^{\ell} M_{a_{i}, b_{i}}$, we have $\left\|\phi_{\pi}\right\|=\left\|\phi_{\text {ker } \pi}\right\|$ and $\left\|\phi_{\pi}\right\|_{c b}=\left\|\phi_{\text {ker } \pi}\right\|_{c b}$ where for irreducible representation $\pi: A \rightarrow \mathcal{B}\left(H_{\pi}\right), \phi_{\pi}=\sum_{i=1}^{\ell} M_{\pi\left(a_{i}\right), \pi\left(b_{i}\right)} \in \mathcal{E} \ell\left(\mathcal{B}\left(H_{\pi}\right)\right)$ (as in [36, §4]).

Theorem 2.2 (Timoney). For $A$ a $C^{*}$-algebra and arbitrary $\phi \in \mathcal{E} \ell(A)$ of length $\ell$ we have

$$
\|\phi\|_{c b}=\left\|\phi^{(\ell)}\right\| \leq \sqrt{\ell}\|\phi\|
$$

where $\phi^{(\ell)}$ denotes the $\ell$-th amplification of $\phi$ on $M_{\ell}(A), \phi^{(\ell)}:\left[x_{i, j}\right] \mapsto\left[\phi\left(x_{i, j}\right)\right]$.

In particular, on each $\mathcal{E} \ell_{\ell}(A)$ the metric induced by the $c b$-norm is equivalent to the metric induced by the operator norm.

\section{Two-Sided MUlTipliCATIONS ON PRIME $C^{*}$-ALGEBRAS}

If $A$ is a prime $C^{*}$-algebra, we prove here (Theorem 3.4) that $\operatorname{TM}(A)$ and $\operatorname{TM}_{0}(A)$ must be closed in $\mathcal{B}(A)$.

The crucial step is the following lemma.

Lemma 3.1. Let $a, b, c$ and $d$ be norm-one elements of an operator space $V$. If

$$
\|a \otimes b-c \otimes d\|_{h}<\varepsilon \leq 1 / 3
$$


then there exists a complex number $\mu$ of modulus one such that

$$
\max \{\|a-\mu c\|,\|b-\bar{\mu} d\|\}<6 \varepsilon .
$$

Proof. First we dispose of the simpler cases where $a$ and $c$ are linearly dependent or where $b$ and $d$ are linearly dependent. If $a$ and $c$ are dependent then $a=\mu c$ with $|\mu|=1$. So $a \otimes b-c \otimes d=c \otimes(\mu b-d)$ and $\|a \otimes b-c \otimes d\|_{h}=\|\mu b-d\|<\varepsilon<6 \varepsilon$. Similarly if $b$ and $d$ are dependent, $b=\bar{\mu} d$ with $|\mu|=1$ and $\|\bar{\mu} a-c\|<\varepsilon$.

Leaving aside these cases, $a \otimes b-c \otimes d$ is a tensor of rank 2. By [5] Lemma 2.3] there is an invertible matrix $S \in \mathbb{M}_{2}$ such that

$$
\left\|\left[\begin{array}{ll}
a & -c
\end{array}\right] S\right\|<\varepsilon \text { and }\left\|S^{-1}\left[\begin{array}{l}
b \\
d
\end{array}\right]\right\|<\varepsilon .
$$

Write $\alpha_{i, j}$ for the $i, j$ entry of $S$ and $\beta_{i, j}$ for the $i, j$ entry of $S^{-1}$.

Since $\alpha_{1,1} \beta_{1,1}+\alpha_{1,2} \beta_{2,1}=1$, at least one of the absolute values $\left|\alpha_{1,1}\right|,\left|\beta_{1,1}\right|$, $\left|\alpha_{1,2}\right|$ or $\left|\beta_{2,1}\right|$ must be at least $1 / \sqrt{2}$. We treat the four cases separately, by very similar arguments.

$\left|\alpha_{1,1}\right| \geq 1 / \sqrt{2}$ : Now from

$$
\left[\begin{array}{ll}
a & -c
\end{array}\right] S=\left[\alpha_{1,1} a-\alpha_{2,1} c \quad \alpha_{1,2} a-\alpha_{2,2} c\right]
$$

we have $\left\|\alpha_{1,1} a-\alpha_{2,1} c\right\|<\varepsilon$, so

$$
\left\|a-\frac{\alpha_{2,1}}{\alpha_{1,1}} c\right\|<\frac{\varepsilon}{\left|\alpha_{1,1}\right|} \leq \sqrt{2} \varepsilon .
$$

Let $\lambda=\alpha_{2,1} / \alpha_{1,1}$. Then

$$
|1-| \lambda||=|\|a\|-| \lambda|\|c|| \mid \leq\| a-\lambda c \| \leq \sqrt{2} \varepsilon,
$$

and so $|\lambda| \in[1-\sqrt{2} \varepsilon, 1+\sqrt{2} \varepsilon]$. Also

$$
\left|\frac{\lambda}{|\lambda|}-\lambda\right|=|\lambda| \frac{|1-| \lambda||}{|\lambda|} \leq(1+\sqrt{2} \varepsilon) \frac{\sqrt{2} \varepsilon}{1-\sqrt{2} \varepsilon}<(\sqrt{2}+2) \varepsilon
$$

(since $\varepsilon \leq 1 / 3$ ). So for $\mu=\frac{\lambda}{|\lambda|}$ we have $|\mu|=1$ and

$$
\|a-\mu c\| \leq\|a-\lambda c\|+|\lambda-\mu|\|c\|<\sqrt{2} \varepsilon+(\sqrt{2}+2) \varepsilon<5 \varepsilon .
$$

Then

$$
\begin{aligned}
a \otimes b-c \otimes d & =a \otimes b-(\mu c \otimes \bar{\mu} d) \\
& =(a \otimes b)-(a \otimes \bar{\mu} d)+(a \otimes \bar{\mu} d)-(\mu c \otimes \bar{\mu} d) \\
& =(a \otimes(b-\bar{\mu} d))+((a-\mu c) \otimes \bar{\mu} d)
\end{aligned}
$$

and thus

$$
\begin{aligned}
\|b-\bar{\mu} d\| & =\|a \otimes(b-\bar{\mu} d)\|_{h} \\
& \leq\|a \otimes b-c \otimes d\|_{h}+\|(a-\mu c) \otimes \bar{\mu} d\|_{h} \\
& <\varepsilon+5 \varepsilon=6 \varepsilon .
\end{aligned}
$$

$\left|\alpha_{1,2}\right| \geq 1 / \sqrt{2}$ : We start now with $\left\|\alpha_{1,2} a-\alpha_{2,2} c\right\|<\varepsilon$ and proceed in the same way (with $\lambda=\alpha_{2,2} / \alpha_{1,2}$ ). 
$\left|\beta_{1,1}\right| \geq 1 / \sqrt{2}$ : In this case we use

$$
S^{-1}\left[\begin{array}{l}
b \\
d
\end{array}\right]=\left[\begin{array}{l}
\beta_{1,1} b+\beta_{1,2} d \\
\beta_{2,1} b+\beta_{2,2} d
\end{array}\right]
$$

and $\left\|\beta_{1,1} b+\beta_{1,2} d\right\|<\varepsilon$, leading to a similar argument (with $\lambda=-\beta_{1,2} / \beta_{1,1}$ and $b$ taking the role of $a$ ).

$\left|\beta_{2,1}\right| \geq 1 / \sqrt{2}$ : In this case use $\left\|\beta_{2,1} b+\beta_{2,2} d\right\|<\varepsilon$.

Corollary 3.2. If $V$ is an operator space, the set $S_{1}=\{a \otimes b: a, b \in V\}$ of all elementary tensors forms a closed subset of $V \otimes_{h} V$.

Proof. Suppose $a_{n} \otimes b_{n} \rightarrow u \in V \otimes_{h} V$ (for $a_{n}, b_{n} \in V$ ). If $u=0$, certainly $u \in S_{1}$ and otherwise we may assume $\|u\|_{h}=1$ and also that

$$
\left\|a_{n} \otimes b_{n}\right\|_{h}=1=\left\|a_{n}\right\|=\left\|b_{n}\right\| \quad(n \geq 1) .
$$

Passing to a subsequence, we may suppose

$$
\left\|a_{n} \otimes b_{n}-a_{n+1} \otimes b_{n+1}\right\|_{h} \leq \frac{1}{6 \cdot 2^{n}} \quad(n \geq 1) .
$$

By Lemma 3.1. we may multiply $a_{n}$ and $b_{n}$ by complex conjugate modulus one scalars chosen inductively to get $a_{n}^{\prime}$ and $b_{n}^{\prime}$ such that

$$
a_{n} \otimes b_{n}=a_{n}^{\prime} \otimes b_{n}^{\prime}, \quad\left\|a_{n}^{\prime}-a_{n+1}^{\prime}\right\| \leq 1 / 2^{n} \quad \text { and } \quad\left\|b_{n}^{\prime}-b_{n+1}^{\prime}\right\| \leq 1 / 2^{n} \quad(n \geq 1) .
$$

In this way we find $a=\lim _{n \rightarrow \infty} a_{n}^{\prime}$ and $b=\lim _{n \rightarrow \infty} b_{n}^{\prime}$ in $V$ with $u=a \otimes b \in S_{1}$.

Question 3.3. If $V$ is an operator space and $\ell>1$, is the set

$$
S_{\ell}=\left\{\sum_{i=1}^{\ell} a_{i} \otimes b_{i}: a_{i}, b_{i} \in V\right\}
$$

of all tensors of rank at most $\ell$ closed in $V \otimes_{h} V$ ? In particular, can we extend Lemma 3.1 as follows.

Let $V$ be an operator space and let $\mathbf{a} \odot \mathbf{b}$ and $\mathbf{c} \odot \mathbf{d}$ be two norm-one tensors of the same (finite) rank $\ell$ in $V \otimes_{h} V$, where a, c and $\mathbf{b}, \mathbf{d}$ are, respectively, $1 \times \ell$ and $\ell \times 1$ matrices with entries in $V$. Suppose that $\|\mathbf{a} \odot \mathbf{b}-\mathbf{c} \odot \mathbf{d}\|_{h}<\varepsilon$ for some $\varepsilon>0$. Can we find absolute constants $C$ and $\delta$ (which depend only on $\ell$ and $\varepsilon$ ) so that $\delta \rightarrow 0$ as $\varepsilon \rightarrow 0$ with the following property:

There exists an invertible matrix $S \in \mathbb{M}_{\ell}$ such that

$$
\|S\|,\left\|S^{-1}\right\| \leq C, \quad\left\|\mathbf{a} S^{-1}-\mathbf{c}\right\|<\delta \quad \text { and } \quad\|S \mathbf{b}-\mathbf{d}\|<\delta ?
$$

Theorem 3.4. If $A$ is a prime $C^{*}$-algebra, then both $\operatorname{TM}(A)$ and $\operatorname{TM}_{0}(A)$ are norm closed.

Proof. By Theorem 2.2 we may work with the cb-norm instead of the (operator) norm. Since $A$ is prime, by Mathieu's theorem (Theorem 2.1) the canonical map $\Theta: M(A) \otimes_{h} M(A) \rightarrow \operatorname{ICB}(A), \Theta: a \otimes b \mapsto M_{a, b}$, is isometric. By Corollary 3.2 the set $S_{1}$ of all elementary tensors in $M(A) \otimes_{h} M(A)$ is closed in the Haagerup norm. Therefore, $\operatorname{TM}(A)=\Theta(S)$ is closed in the cb-norm.

For the case of $\operatorname{TM}_{0}(A)$, we use the same argument but work with the restriction of $\Theta$ to $A \otimes_{h} A$.

Corollary 3.5. If $A$ is a prime $C^{*}$-algebra, then the sets $\operatorname{TM}_{c p}(A), \operatorname{InnAut}_{a l g}(A)$, and $\operatorname{InnAut}(A)$ (see (2.3)) are all norm closed. 
Proof. Suppose that an operator $\phi$ in the norm closure of any of these sets. Then, by Theorem 3.4 there are $b, c \in M(A)$ such that $\phi=M_{b, c}$. Let $\varepsilon>0$.

Suppose that $\phi$ is in the closure of $\operatorname{TM}_{c p}(A)$. By Theorem 2.2 we may work with the cb-norm instead of the (operator) norm. We may also assume that $\|\phi\|_{c b}=1=$ $\|b\|=\|c\|$. Then there is $a \in M(A)$ such that

$$
\left\|M_{b, c}-M_{a, a^{*}}\right\|_{c b}=\left\|b \otimes c-a \otimes a^{*}\right\|_{h}<\varepsilon
$$

(Theorem 2.1). If $\varepsilon \leq 1 / 3$, by Lemma 3.1 we can find a complex number $\mu$ of modulus one such that $\|b-\mu a\|<6 \varepsilon$ and $\left\|c-\bar{\mu} a^{*}\right\|<6 \varepsilon$. Then $\left\|b-c^{*}\right\| \leq 12 \varepsilon$. Hence $c=b^{*}$, so $\phi=M_{b, c} \in \mathrm{TM}_{c p}(A)$.

Suppose that $\phi$ is in the closure of $\operatorname{InnAutalg}_{\text {alg }}(A)$. Then there is an invertible element $a \in M(A)$ such that $\left\|M_{b, c}-M_{a, a^{-1}}\right\|<\varepsilon$. Since $A$ is an essential ideal in $M(A)$, this implies $\left\|b x c-a x a^{-1}\right\|<\varepsilon$ for all $x \in M(A),\|x\| \leq 1$. Letting $x=1$ we obtain $\|b c-1\|<\varepsilon$. Hence $c=b^{-1}$, so $\phi=M_{b, c} \in \operatorname{InnAutalg}_{\text {alg }}(A)$.

$\operatorname{Inn} A u t(A)$ is norm closed as an intersection $\operatorname{TM}_{c p}(A) \cap \operatorname{Inn} A u t_{a l g}(A)$ of two closed sets.

\section{On homogeneous $C^{*}$-Algebras}

We recall that a $C^{*}$-algebra $A$ is called $n$-homogeneous (where $n$ is finite) if every irreducible representation of $A$ acts on an $n$-dimensional Hilbert space. We say that $A$ is homogeneous if it is $n$-homogeneous for some $n$. We will use the following definitions and facts about homogeneous $C^{*}$-algebras:

Remark 4.1. Let $A$ be an $n$-homogeneous $C^{*}$-algebra. By [20, Theorem 4.2] $\operatorname{Prim}(A)$ is a (locally compact) Hausdorff space. If there is no danger of confusion, we simply write $X$ for $\operatorname{Prim}(A)$.

(a) A well-known theorem of Fell [10, Theorem 3.2], and Tomiyama-Takesaki 37. Theorem 5] asserts that for any $n$-homogeneous $C^{*}$-algebra, $A$, there is a locally trivial bundle $\mathcal{E}$ over $X$ with fibre $\mathbb{M}_{n}$ and structure group $P U(n)=\operatorname{Aut}\left(\mathbb{M}_{n}\right)$ such that $A$ is isomorphic to the $C^{*}$-algebra $\Gamma_{0}(\mathcal{E})$ of continuous sections of $\mathcal{E}$ which vanish at infinity.

Moreover, any two such algebras $A_{i}=\Gamma_{0}\left(\mathcal{E}_{i}\right)$ with primitive spectra $X_{i}$ $(i=1,2)$ are isomorphic if and only if there is a homeomorphism $f: X_{1} \rightarrow$ $X_{2}$ such that $\mathcal{E}_{1} \cong f^{*}\left(\mathcal{E}_{2}\right)$ (the pullback bundle) as bundles over $X_{1}$ (see [37. Theorem 6]). Thus, we may identify $A$ with $\Gamma_{0}(\mathcal{E})$.

(b) For $a \in A$ and $t \in X$ we define $\pi_{t}(a)=a(t)$. Then, after identifying the fibre $\mathcal{E}_{t}$ with $\mathbb{M}_{n}, \pi_{t}: a \mapsto \pi_{t}(a)$ (for $t \in X$ ) gives all irreducible representations of $A$ (up to the equivalence).

For a closed subset $S \subseteq X$ we define

$$
I_{S}=\bigcap_{t \in S} \operatorname{ker} \pi_{t}=\{a \in A: a(t)=0 \text { for all } t \in S\} .
$$

By [11, VII 8.7.] any closed two-sided ideal of $A$ is of the form $I_{S}$ for some closed subset $S \subset X$. Further, by the the generalized Tietze Extension Theorem we may identify $A_{S}=A / I_{S}$ with $\Gamma_{0}\left(\left.\mathcal{E}\right|_{S}\right)$ (see [11, II. 14.8. and VII 8.6.]). If $S=\{t\}$ we just write $A_{t}$.

(c) If $\phi \in \operatorname{IB}(A)$ and $S \subset X$ closed, we write $\phi_{S}$ for the operator $\phi_{I_{S}}$ on $A_{S}$ (see (2.1)). If $S=\{t\}$ we just write $\phi_{t}$. If $A$ is trivial (i.e. $A=C_{0}\left(X, \mathbb{M}_{n}\right)$ ), 
we will consider $\phi_{t}$ as an operator $: \mathbb{M}_{n} \rightarrow \mathbb{M}_{n}$ (after identifying $A_{t}$ with $\mathbb{M}_{n}$ in the obvious way).

If $U \subset X$ is open, we can regard $B=\Gamma_{0}\left(\left.\mathcal{E}\right|_{U}\right)$ as the ideal $I_{X \backslash U}$ of $A$ (by extending sections to be zero outside $U$ ) and for $\phi \in \operatorname{IB}(A)$, we then have a restriction $\left.\phi\right|_{U} \in \operatorname{IB}(B)$ of $\phi$ to this ideal (with $\left(\left.\phi\right|_{U}\right)_{t}=\phi_{t}$ for $t \in U$ ).

(d) $\operatorname{IB}(A)=\operatorname{ICB}(A)$. Indeed, for $\phi \in \operatorname{IB}(A)$ and $t \in X$ we have $\left\|\phi_{t}\right\|_{c b} \leq n\left\|\phi_{t}\right\|$ (29, p. 114]), so by (2.2) we have $\|\phi\|_{c b} \leq n\|\phi\|$. Hence $\phi \in \operatorname{ICB}(A)$.

(e) Since each $a \in Z(A)$ has $a(t)$ a multiple of the identity in the fibre $\mathcal{E}_{t}$ for each $t \in X$, we can identify $Z(A)$ with $C_{0}(X)$. Observe that $A$ is quasicentral (i.e. no primitive ideal of $A$ contains $Z(A)$ ).

(f) By [25, Lemma 3.2] we can identify $M(A)$ with $\Gamma_{b}(\mathcal{E})$ (the $C^{*}$-algebra of bounded continuous sections of $\mathcal{E})$. As usual, we will identify $Z(M(A))$ with $C_{b}(X)$ (using the Dauns-Hofmann theorem [33, Theorem A.34]).

If $A=C_{0}\left(X, \mathbb{M}_{n}\right)$, it is well known that $M(A)=C_{b}\left(X, \mathbb{M}_{n}\right)=C\left(\beta X, \mathbb{M}_{n}\right)$ 1. Corollary 3.4], where $\beta X$ denotes the Stone-Čech compactification.

(g) On each fibre $\mathcal{E}_{t}$ we can introduce an inner product $\langle\cdot, \cdot\rangle_{2}$ as follows.

Choose an open covering $\left\{U_{\alpha}\right\}$ of $X$ such that each $\left.\mathcal{E}\right|_{U_{\alpha}}$ is isomorphic to $U_{\alpha} \times \mathbb{M}_{n}$ (as an $\mathbb{M}_{n}$-bundle), say via isomorphism $\Phi_{\alpha}$. Let

$$
\langle\xi, \eta\rangle_{2}=\operatorname{tr}\left(\Phi_{\alpha}(\xi) \Phi_{\alpha}(\eta)^{*}\right) \quad\left(\xi, \eta \in \mathcal{E}_{t}\right),
$$

where $\alpha$ is chosen so that $t \in U_{\alpha}$ and $\operatorname{tr}(\cdot)$ is the standard trace on $\mathbb{M}_{n}$. This is independent of the choice of $\alpha$ since all automorphisms of $\mathbb{M}_{n}$ are inner and $\operatorname{tr}(\cdot)$ is invariant under conjugation by unitaries. If $a, b \in M(A)=$ $\Gamma_{b}(\mathcal{E})$ then $t \mapsto\langle a(t), b(t)\rangle_{2}$ is in $C_{b}(X)$.

The norm $\|\cdot\|_{2}$ on $\mathcal{E}_{t}$ associated with $\langle\cdot, \cdot\rangle_{2}$ satisfies

$$
\|\xi\| \leq\|\xi\|_{2} \leq \sqrt{n}\|\xi\| \quad\left(\xi \in \mathcal{E}_{t}\right)
$$

In the terminology of $\left[\underline{8},\left(\mathcal{E},\langle\cdot, \cdot\rangle_{2}\right)\right.$ is a (complex continuous) Hilbert bundle of rank $n^{2}$ with fibre norms equivalent to the original $C^{*}$-norms (by (4.2)).

(h) $A$ is said to have the finite type property if $\mathcal{E}$ can be trivialized over some finite open cover of $X$. By [25, Remark 3.3] $M(A)$ is homogeneous if and only if $A$ has the finite type property. When this fails, it is possible to have $\operatorname{Prim}(M(A))$ non-Hausdorff [4, Theorem 2.1]. On the other hand, $M(A)$ is always quasi-standard (see [3, Corollary 4.10]).

For completeness we include a proof of the following.

Proposition 4.2. Let $X$ be a locally compact Hausdorff space and $A=C_{0}\left(X, \mathbb{M}_{n}\right)$.

(a) $\operatorname{IB}(A)$ can be identified with $C_{b}\left(X, \mathcal{B}\left(\mathbb{M}_{n}\right)\right)$ by a mapping which sends an operator $\phi \in \operatorname{IB}(A)$ to the function $\left(t \mapsto \phi_{t}\right)$.

(b) Any $\phi \in \operatorname{IB}(A)$ can be written in the form

$$
\phi=\sum_{k, i=1}^{n} M_{e_{k, i}, a_{k, i}},
$$

where $\left(e_{k, i}\right)_{k, i=1}^{n}$ are standard matrix units of $\mathbb{M}_{n}$ (considered as constant functions in $\left.C_{b}\left(X, \mathbb{M}_{n}\right)=M(A)\right)$ and $a_{k, i} \in M(A)$ depend on $\phi$.

Thus, we have

$$
\operatorname{IB}(A)=\operatorname{ICB}(A)=C_{b}\left(X, \mathcal{B}\left(\mathbb{M}_{n}\right)\right)=\mathcal{E} \ell(A)=\mathcal{E} \ell_{n^{2}}(A) .
$$


Proof. Let $\phi \in \operatorname{IB}(A)$.

(a) Suppose that the function $t \mapsto \phi_{t}: X \rightarrow \mathcal{B}\left(\mathbb{M}_{n}\right)$ is discontinuous at some point $t_{0} \in X$. Then there is a net $\left(t_{\alpha}\right)$ in $X$ converging to $t_{0}$ such that $\left\|\phi_{t_{\alpha}}-\phi_{t_{0}}\right\| \geq$ $\delta>0$ for all $\alpha$. So there is $u_{\alpha} \in \mathbb{M}_{n}$ of norm at most 1 with $\left\|\phi_{t_{\alpha}}\left(u_{\alpha}\right)-\phi_{t_{0}}\left(u_{\alpha}\right)\right\| \geq \delta$. Passing to a subnet we may suppose $u_{\alpha} \rightarrow u$ and then (since $\left\|\phi_{t_{\alpha}}\right\| \leq\|\phi\|$ and $\left.\left\|\phi_{t_{0}}\right\| \leq\|\phi\|\right)$ we must have

$$
\left\|\phi_{t_{\alpha}}(u)-\phi_{t_{0}}(u)\right\|>\delta / 2
$$

for $\alpha$ large enough.

Now choose $f \in C_{0}(X)$ equal to 1 on a neighbourhood of $t_{0}$ and put $a(t)=f(t) u$. We then have $a \in A$ and

$$
\pi_{t_{\alpha}}(\phi(a))=f\left(t_{\alpha}\right) \phi_{t_{\alpha}}(u)=\phi_{t_{\alpha}}(u)
$$

for large $\alpha$ and this contradicts continuity of $\phi(a)$ at $t_{0}$.

So $t \mapsto \phi_{t}$ must be continuous (and also bounded by $\|\phi\|$ ).

Conversely, assume that the function $t \mapsto \phi_{t}$ is continuous and uniformly bounded by some $M>0$. Then for $a \in A, t \mapsto \phi_{t}\left(\pi_{t}(a)\right)$ is continuous, bounded and vanishes at infinity, hence in $A$. So there is an associated mapping $\phi: A \rightarrow A$ which is easily seen to be bounded and linear. Moreover $\phi \in \operatorname{IB}(A)$ since all ideals of $A$ are of the form $I_{S}$ for some closed $S \subset X$.

(b) First assume that $A$ is unital, so that $X$ is compact. Then each $x \in A$ is a linear combination over $C(X)=Z(A)$ of the $e_{i, j}$ and since $\phi$ is $C(X)$-linear, we have

$$
x=\sum_{i, j=1}^{n} x_{i, j} e_{i, j} \Rightarrow \phi(x)=\sum_{i, j=1}^{n} x_{i, j} \phi\left(e_{i, j}\right) .
$$

We may write

$$
\phi\left(e_{i, j}\right)=\sum_{k, \ell=1}^{n} \phi_{i, j, k, \ell} e_{k, \ell}=\sum_{k=1}^{n} e_{k, i} e_{i, j}\left(\sum_{\ell=1}^{n} \phi_{i, j, k, \ell} e_{j, \ell}\right)
$$

where $\phi_{i, j, k, \ell} \in C(X)$. It follows that

$$
\phi(x)=\sum_{k, i=1}^{n} e_{k, i} x\left(\sum_{j, \ell=1}^{n} \phi_{i, j, k, \ell} e_{j, \ell}\right) \Rightarrow \phi=\sum_{k, i=1}^{n} M_{e_{k, i}, a_{k, i}}
$$

where $a_{k, i}=\sum_{j, \ell=1}^{n} \phi_{i, j, k, \ell} e_{j, \ell} \in M(A)$.

Now suppose that $A$ is non-unital (so that $X$ is non-compact). By (a) we can identify $\phi$ with the function $t \mapsto \phi_{t}: X \rightarrow \mathcal{B}\left(\mathbb{M}_{n}\right)$, which can be then uniquely extended to a continuous function $\beta X \rightarrow \mathcal{B}\left(\mathbb{M}_{n}\right)$. This extension defines an operator in $\operatorname{IB}\left(C\left(\beta X, \mathbb{M}_{n}\right)\right)=\operatorname{IB}(M(A))$, which we also denote by $\phi$. By the first part of the proof, $\phi$ can be represented as (4.3).

Remark 4.3. In fact, in the case of general separable $C^{*}$-algebras $A$, Magajna [25] establishes the equivalence of the following properties:

(a) $\operatorname{IB}(A)=\mathcal{E} \ell(A)$.

(b) $\mathcal{E} \ell(A)$ is norm dense in $\operatorname{IB}(A)$.

(c) $A$ is a finite direct sums of homogeneous $C^{*}$-algebras with the finite type property. 
Analyzing the arguments in [25], for the implication (c) $\Rightarrow$ (a) it is sufficient to assume that $X$ is paracompact.

Since any $n$-homogeneous $C^{*}$-algebra is locally of the form $C\left(K, \mathbb{M}_{n}\right)$ for some compact subset $K$ of $X$ with $K^{\circ} \neq \emptyset$, we have the following consequence of Proposition 4.2

Corollary 4.4. If $A$ is a homogeneous $C^{*}$-algebra, then for any $\phi \in \operatorname{IB}(A)$ the function $t \mapsto\left\|\phi_{t}\right\|$ is continuous on $X$. Hence the cozero set $\operatorname{coz}(\phi)=\{t \in X$ : $\left.\phi_{t} \neq 0\right\}$ is $\sigma$-compact and open in $X$.

\section{Fibrewise Length Restrictions}

Here we consider a homogeneous $C^{*}$-algebra $A=\Gamma_{0}(\mathcal{E})$ and operators $\phi \in \operatorname{IB}(A)$ such that $\phi_{t}$ is a two-sided multiplication on each fibre $A_{t}$ (with $t \in X$, and $X=$ $\operatorname{Prim}(A)$ as usual). We will write $\phi \in \operatorname{IB}_{1}(A)$ for this hypothesis. For separable $A$, the main result in this section (Theorem 5.15) characterizes when all such operators $\phi$ are two-sided multiplications, in terms of triviality of complex line subbundles of $\left.\mathcal{E}\right|_{U}$ for $U \subset X$ open.

In addition to $\operatorname{IB}_{1}(A)$, we introduce various subsets $\operatorname{IB}_{1}^{\mathrm{nv}}(A)$ and $\operatorname{IB}_{0,1}(A)$ (Notation (5.5) which are designed to facilitate the description of $\operatorname{TM}(A), \operatorname{TM}_{0}(A)$ and both of their norm closures in terms of complex line bundles. The sufficient condition that ensures $\operatorname{IB}_{1}^{\mathrm{nv}}(A) \subset \mathrm{TM}(A)$ is that $X$ is paracompact with vanishing second integral Čech cohomology group $\check{H}^{2}(X ; \mathbb{Z})$ (Corollary 5.11). For $X$ compact of finite covering dimension $d$ and $A=C\left(X, \mathbb{M}_{n}\right)$ we show that $\operatorname{TM}(A) \subsetneq \operatorname{IB}_{1}(A)$ provided $\check{H}^{2}(X ; \mathbb{Z}) \neq 0$ and $n^{2} \geq(d+1) / 2$ (Proposition 5.12). We get the same conclusion $\operatorname{TM}(A) \subsetneq \operatorname{IB}_{1}(A)$ for $\sigma$-unital $n$-homogeneous $C^{*}$-algebras $A=\Gamma_{0}(\mathcal{E})$ with $n \geq 2$ provided $X$ has a nonempty open subset homeomorphic to (an open set in) $\mathbb{R}^{d}$ with $d \geq 3$ (Corollary 5.16).

Notation 5.1. Let $A$ be an $n$-homogeneous $C^{*}$-algebra. For $\ell \geq 1$ we write

$$
\operatorname{IB}_{\ell}(A)=\left\{\phi \in \operatorname{IB}(A): \phi_{t} \in \mathcal{E} \ell_{\ell}\left(A_{t}\right) \text { for all } t \in X\right\} .
$$

Lemma 5.2. Let $A=\Gamma_{0}(\mathcal{E})$ be a homogeneous $C^{*}$-algebra and $\phi \in \operatorname{IB}_{\ell}(A)$.

If $t_{0} \in X$ is such that $\phi_{t_{0}} \in \mathcal{E} \ell_{\ell}\left(A_{t_{0}}\right) \backslash \mathcal{E} \ell_{\ell-1}\left(A_{t_{0}}\right)$ (that is, such that $\phi_{t_{0}}$ has length exactly the maximal $\ell)$, then there are $a_{1}, \ldots, a_{\ell}, b_{1}, \ldots, b_{\ell} \in A$ and a compact neighbourhood $N$ of $t_{0}$ such that $\phi$ agrees with the elementary operator $\sum_{i=1}^{\ell} M_{a_{i}, b_{i}}$ modulo the ideal $I_{N}$, that is

$$
\phi(x)-\sum_{i=1}^{\ell} a_{i} x b_{i} \in I_{N} \quad \text { for all } x \in A .
$$

Moreover, we can choose $N$ so that $\phi_{t} \in \mathcal{E} \ell_{\ell}\left(A_{t}\right) \backslash \mathcal{E} \ell_{\ell-1}\left(A_{t}\right)$ for all $t \in N$ (that is, $\phi_{t}$ is of the maximal length $\ell$ for $t$ in a neighbourhood of $\left.t_{0}\right)$.

Proof. Choose a compact neighbourhood $K$ of $t_{0}$ such that $A_{K} \cong C\left(K, \mathbb{M}_{n}\right)$ and let $\phi_{K}$ be the induced operator (Remark $4.1(\mathrm{~b}),(\mathrm{c})$ ).

Then, for $x \in A_{K}$ we have $\phi_{K}(x)=\sum_{i=1}^{n^{2}} c_{i} x d_{i}$ for some $c_{i}, d_{i} \in A_{K}$ (by Proposition $4.2(\mathrm{~b}))$. Moreover we can assume that $\left\{c_{1}(t), \ldots, c_{n^{2}}(t)\right\}$ are linearly independent for each $t \in K$, and even independent of $t$. Since $\left(\phi_{K}\right)_{t_{0}}=\phi_{t_{0}}$ has 
length $\ell$, we must be able to write (in $\mathbb{M}_{n} \otimes \mathbb{M}_{n}$ )

$$
\sum_{i=1}^{n^{2}} c_{i}\left(t_{0}\right) \otimes d_{i}\left(t_{0}\right)=\sum_{j=1}^{\ell} c_{j}^{\prime} \otimes d_{j}^{\prime} .
$$

We can choose $d_{1}^{\prime}, \ldots, d_{\ell}^{\prime}$ to be a maximal linearly independent subsequence of $d_{1}\left(t_{0}\right), \ldots, d_{n^{2}}\left(t_{0}\right)$. Then, via elementary linear algebra, there is a matrix $\alpha$ of size $n^{2} \times \ell$ and another matrix $\beta$ of size $\ell \times n^{2}$ so that

$$
\left[\begin{array}{c}
d_{1}\left(t_{0}\right) \\
\vdots \\
d_{n^{2}}\left(t_{0}\right)
\end{array}\right]=\alpha\left[\begin{array}{c}
d_{1}^{\prime} \\
\vdots \\
d_{\ell}^{\prime}
\end{array}\right], \quad\left[\begin{array}{c}
d_{1}^{\prime} \\
\vdots \\
d_{\ell}^{\prime}
\end{array}\right]=\beta\left[\begin{array}{c}
d_{1}\left(t_{0}\right) \\
\vdots \\
d_{n^{2}}\left(t_{0}\right)
\end{array}\right]
$$

and $\beta \alpha$ the identity. We have

$$
\left[\begin{array}{lll}
c_{1}^{\prime} & \cdots & c_{\ell}^{\prime}
\end{array}\right]=\left[\begin{array}{lll}
c_{1}\left(t_{0}\right) & \cdots & c_{n^{2}}\left(t_{0}\right)
\end{array}\right] \alpha .
$$

If we define

$$
\left[\begin{array}{c}
d_{1}^{\prime}(t) \\
\vdots \\
d_{\ell}^{\prime}(t)
\end{array}\right]=\beta\left[\begin{array}{c}
d_{1}(t) \\
\vdots \\
d_{n^{2}}(t)
\end{array}\right]
$$

then $d_{1}^{\prime}(t), \ldots, d_{\ell}^{\prime}(t)$ must be linearly independent for all $t$ in some compact neighbourhood $N$ of $t_{0}$. Thus for $t \in N$ we have (in $\mathbb{M}_{n} \otimes \mathbb{M}_{n}$ )

$$
\sum_{i=1}^{n^{2}} c_{i}(t) \otimes d_{i}(t)=\sum_{i=1}^{n^{2}} c_{i}\left(t_{0}\right) \otimes d_{i}(t)=\sum_{j=1}^{\ell} c_{j}^{\prime} \otimes d_{j}^{\prime}(t) .
$$

By Remark4.1 (b) we can find elements $a_{j}, b_{j} \in A(1 \leq j \leq \ell)$ such that $a_{j}(t)=c_{j}^{\prime}$ and $b_{j}(t)=d_{j}^{\prime}(t)$ for all $t \in N$.

Since for each $t \in N$ both of the sets $\left\{a_{1}(t), \ldots, a_{\ell}(t)\right\}$ and $\left\{b_{1}(t), \ldots, b_{\ell}(t)\right\}$ are linearly independent, we get that $\phi_{t}=\sum_{j=1}^{\ell} M_{a_{j}(t), b_{j}(t)}$ has length exactly $\ell$ for all $t \in N$ as required.

Corollary 5.3. Let $A$ be a homogeneous $C^{*}$-algebra and $\phi \in \mathrm{IB}_{1}(A)$. If $t_{0} \in X$ is such that $\phi_{t_{0}} \neq 0$ then there is a compact neighbourhood $N$ of $t_{0}$ and $a, b \in A$ such that $a(t) \neq 0$ and $b(t) \neq 0$ for all $t \in N$ and $\phi$ agrees with $M_{a, b}$ modulo the ideal $I_{N}$.

Remark 5.4. Let $A=\Gamma_{0}(\mathcal{E})$ be a homogeneous $C^{*}$-algebra, $a, b \in M(A)=\Gamma_{b}(\mathcal{E})$ and $\phi=M_{a, b}$.

We may replace $a$ and $b$ by

$$
t \mapsto \sqrt{\frac{\|b(t)\|}{\|a(t)\|}} a(t) \quad \text { and } \quad t \mapsto \sqrt{\frac{\|a(t)\|}{\|b(t)\|}} b(t)
$$

without changing $\phi$ so as to ensure that $\|a(t)\|=\|b(t)\|$ for each $t \in X$ and that $\left\|\phi_{t}\right\|=\|a(t)\|^{2}=\|b(t)\|^{2}$ for $t \in X$.

Notation 5.5. Let $A$ be a homogeneous $C^{*}$-algebra. We write

$$
\mathrm{IB}_{1}^{\mathrm{nv}}(A)=\left\{\phi \in \operatorname{IB}(A): 0 \neq \phi_{t} \in \operatorname{TM}\left(A_{t}\right) \text { for all } t \in X\right\}
$$

(where nv signifies nowhere-vanishing). 
We also use

$$
\begin{aligned}
\operatorname{IB}_{0}(A) & =\left\{\phi \in \operatorname{IB}(A):\left(t \mapsto\left\|\phi_{t}\right\|\right) \in C_{0}(X)\right\}, \\
\operatorname{IB}_{0,1}(A) & =\operatorname{IB}_{0}(A) \cap \operatorname{IB}_{1}(A), \\
\operatorname{IB}_{0,1}^{\text {nv }}(A) & =\mathrm{IB}_{1}^{\text {nv }}(A) \cap \operatorname{IB}_{0}(A), \text { and } \\
\operatorname{TM}^{\text {nv }}(A) & =\operatorname{TM}(A) \cap \operatorname{IB}_{1}^{\text {nv }}(A) .
\end{aligned}
$$

By Remark 5.4. $\operatorname{TM}_{0}(A)=\operatorname{TM}(A) \cap \operatorname{IB}_{0}(A)$.

Proposition 5.6. Let $A=\Gamma_{0}(\mathcal{E})$ be a homogeneous $C^{*}$-algebra and suppose $\phi \in$ $\mathrm{IB}_{1}^{\mathrm{nv}}(A)$. Then there is a canonically associated complex line subbundle $\mathcal{L}_{\phi}$ of $\mathcal{E}$ with the property that

$$
\phi \in \operatorname{TM}(A) \quad \Longleftrightarrow \quad \mathcal{L}_{\phi} \text { is a trivial bundle. }
$$

Proof. By Corollary 5.3 locally $\phi$ is a two-sided multiplication. That is, given $t_{0} \in$ $X$ there is a compact neighbourhood $N$ of $t_{0}$ and $a, b \in A$ such that $\phi_{t}=M_{a(t), b(t)}$ for all $t \in N$.

We define

$$
\mathcal{L}_{\phi} \cap\left(\left.\mathcal{E}\right|_{N}\right) \text { to be }\{(t, \lambda a(t)): t \in N, \lambda \in \mathbb{C}\} .
$$

Then $\mathcal{L}_{\phi}$ is well-defined since if $N^{\prime}$ is another neighbourhood of a possibly different $t_{0}^{\prime} \in X$ and $a^{\prime}, b^{\prime} \in A$ have $\phi_{t}=M_{a^{\prime}(t), b^{\prime}(t)}$ for all $t \in N^{\prime}$, then there is $\mu(t) \in \mathbb{C} \backslash\{0\}$ such that $a^{\prime}(t)=\mu(t) a(t)$ for $t \in N \cap N^{\prime}$.

The definition we gave of $\mathcal{L}_{\phi} \cap\left(\left.\mathcal{E}\right|_{N}\right)$ shows that $\mathcal{L}_{\phi}$ is a locally trivial complex line subbundle of $\mathcal{E}$. The map

$$
: N \times \mathbb{C} \rightarrow \mathcal{L}_{\phi} \cap\left(\left.\mathcal{E}\right|_{N}\right) \quad \text { given by } \quad(t, \lambda) \mapsto(t, \lambda a(t)) .
$$

provides a local trivialization.

If $\phi \in \operatorname{TM}(A)$, then clearly $\mathcal{L}_{\phi}$ is a trivial bundle. Conversely, If $\mathcal{L}_{\phi}$ is a trivial bundle, choose a continuous nowhere vanishing section $s: X \rightarrow \mathcal{L}_{\phi}$. Then for any neighbourhood $N$ as above there is a continuous map $\zeta: N \rightarrow \mathbb{C} \backslash\{0\}$ such that $a(t)=\zeta(t) s(t)$. If we define $s^{\prime}: X \rightarrow \mathcal{E}$ by $s^{\prime}(t)=(1 / \zeta(t)) b(t)$ for $t \in N$, then we have $s, s^{\prime} \in \Gamma(\mathcal{E})$ well-defined and $\phi_{t}(x(t))=s(t) x(t) s^{\prime}(t)$ for all $x \in A$. Normalizing $s$ and $s^{\prime}$ as in Remark 5.4 we get $c, d \in \Gamma_{b}(\mathcal{E})=M(A)$ (Remark 4.1 (f)) with $\phi=M_{c, d}$.

Notation 5.7. If $A=\Gamma_{0}(\mathcal{E})$ is homogeneous and $\phi \in \operatorname{IB}_{1}(A)$, we consider the cozero set $U=\operatorname{coz}(\phi)$ (open by Corollary 4.4) and then, for $B=\Gamma_{0}\left(\left.\mathcal{E}\right|_{U}\right),\left.\phi\right|_{U} \in$ $\mathrm{IB}_{0,1}^{\mathrm{nv}}(B)$ (see Remark $4.1(\mathrm{c})$ ). We occasionally use $\mathcal{L}_{\phi}$ for the subbundle $\mathcal{L}_{\left.\phi\right|_{U}}$ of $\left.\mathcal{E}\right|_{U}$.

Proposition 5.8. Let $A=\Gamma_{0}(\mathcal{E})$ be an n-homogeneous $C^{*}$-algebra such that $X$ is $\sigma$-compact. If $\mathcal{L}$ is a complex line subbundle of $\mathcal{E}$, then there is $\phi \in \operatorname{IB}_{0,1}^{\mathrm{nv}}(A)$ with $\mathcal{L}_{\phi}=\mathcal{L}$.

Proof. Let $\langle\cdot, \cdot\rangle_{2}$ be as in Remark $4.1(\mathrm{~g})$. With respect to this inner product we have a complementary subbundle $\mathcal{L}^{\perp}$ of $\mathcal{E}$ such that $\mathcal{L} \oplus \mathcal{L}^{\perp}=\mathcal{E}$.

Note that, by local compactness, $X$ has a base consisting of $\sigma$-compact open sets. (If $t_{0} \in U \subset X$ with $U$ open, choose a compact neighborhood $N$ of $t_{0}$ contained in $U$ and a function $f \in C_{0}(X)$ supported in $N$ with $f\left(t_{0}\right)=1$. Take $V=\{t \in X:|f(t)|>0\}$. 
Since $X$ is $\sigma$-compact (and since every $\sigma$-compact space is Lindelöf), we can find a countable open cover $\left\{U_{i}\right\}_{i=1}^{\infty}$ of $X$ such that each restriction $\left.\mathcal{E}\right|_{U_{i}}$ is trivial and each $U_{i}$ is $\sigma$-compact. Then we can find $n^{2}$ norm-one sections $\left(e_{j}^{i}\right)_{j=1}^{n^{2}}$ of $\Gamma_{0}\left(\left.\mathcal{E}\right|_{U_{i}}\right) \cong C_{0}\left(U_{i}, \mathbb{M}_{n}\right)$ such that

$$
\operatorname{span}\left\{e_{1}^{i}(t), \cdots, e_{n^{2}}^{i}(t)\right\}=\mathcal{E}_{t} \cong \mathbb{M}_{n} \text { for all } t \in U_{i}
$$

By extending outside $U_{i}$ with 0 we may assume that $e_{j}^{i}$ are globally defined, so that $e_{j}^{i} \in A$. Define $f_{j}^{i}(t)$ as the orthogonal projection of $e_{j}^{i}$ into the fibre $\mathcal{L}_{t}$, so that $f_{j}^{i} \in A$. We define

$$
\phi: A \rightarrow A \quad \text { by } \phi=\sum_{i=1}^{\infty} \frac{1}{2^{i}}\left(\sum_{j=1}^{n^{2}} M_{f_{j}^{i},\left(f_{j}^{i}\right)^{*}}\right) .
$$

Note that $\phi \in \operatorname{IB}_{0}(A)$ as a sum of an absolutely convergent series of operators in $\operatorname{IB}_{0}(A)$ (and $\operatorname{IB}_{0}(A)$ is norm closed). We claim that $\phi \in \operatorname{IB}_{0,1}^{\mathrm{nv}}(A)$ and $\mathcal{L}_{\phi}=\mathcal{L}$. Indeed, for an arbitrary point $t \in X$ choose a norm-one (in $C^{*}$-norm) vector $s \in \mathcal{L}_{t}$. Then there are scalars $\lambda_{j}^{i}$ with $f_{j}^{i}(t)=\lambda_{j}^{i} \cdot s$ and $\left|\lambda_{j}^{i}\right|=\left\|f_{j}^{i}(t)\right\| \leq \sqrt{n}\left\|e_{j}^{i}(t)\right\|=\sqrt{n}$ (by (4.2)). Then

$$
\phi_{t}=\left(\sum_{i=1}^{\infty} \frac{1}{2^{i}}\left(\sum_{j=1}^{n^{2}}\left|\lambda_{j}^{i}\right|^{2}\right)\right) \cdot M_{s, s^{*}}
$$

This shows that $\phi \in \operatorname{IB}_{0,1}^{\mathrm{nv}}(A)$ and that for all $t \in X$ we have $\phi_{t}=M_{a(t), a^{*}(t)}$ for some $a(t) \in \mathcal{L}_{t}$. By the proof of Proposition 5.6 we conclude $\mathcal{L}_{\phi}=\mathcal{L}$.

Remark 5.9. Let $\mathcal{L}$ be a complex line bundle over a locally compact Hausdorff space $X$.

(a) $\mathcal{L}$ is isomorphic to a subbundle of some $\mathbb{M}_{2}$-bundle $\mathcal{E}$. Indeed, let $\mathcal{F}=$ $\mathcal{L} \oplus(X \times \mathbb{C})$. Then $\mathcal{E}=\operatorname{Hom}(\mathcal{F}, \mathcal{F})=\mathcal{F} \otimes \mathcal{F}^{*}$ is an $\mathbb{M}_{2}$-bundle with the desired property (see [32, Example 3.5]).

Further, if $X$ is $\sigma$-compact, then $A=\Gamma_{0}(\mathcal{E})$ (with $\mathcal{E}$ as above) is an example of a 2-homogeneous $C^{*}$-algebra with $\operatorname{Prim}(A)=X$ that allows an operator $\phi \in \operatorname{IB}_{0,1}^{\mathrm{nv}}(A)$ such that $\mathcal{L}_{\phi} \cong \mathcal{L}$ (by Proposition 5.8).

(b) Suppose that $\mathcal{L}$ is a subbundle of a trivial bundle $X \times \mathbb{C}^{m}$. If $p=\lceil\sqrt{m}\rceil$, then for each $n \geq p$ we can regard $\mathcal{L}$ as a subbundle of a trivial matrix bundle $X \times \mathbb{M}_{n}$, using some linear embedding $\mathbb{C}^{m} \hookrightarrow \mathbb{M}_{n}$.

Remark 5.10. If the space $X$ is paracompact, it is well-known that locally trivial complex line bundles over $X$ are classified by the homotopy classes of maps from $X$ to $\mathbb{C} P^{\infty}$ and/or by the elements of the second integral Cech cohomology $\check{H}^{2}(X ; \mathbb{Z})$ (see e.g. [18, Corollary 3.5.6 and Theorem 3.4.7] and [33, Proposition 4.53 and Theorem 4.42].) By [18, we know that complex line bundles over $X$ are pullbacks of the canonical bundle over $\mathbb{C} P^{\infty}$ (via a map from $X$ to $\mathbb{C} P^{\infty}$ ).

In light of Proposition 5.6 and Remark 5.10, for a given a homogeneous $C^{*}$ algebra $A=\Gamma_{0}(\mathcal{E})$ we define a map

$$
\theta: \operatorname{IB}_{1}^{\mathrm{nv}}(A) \rightarrow \check{H}^{2}(X ; \mathbb{Z})
$$

which sends an operator $\phi \in \operatorname{IB}_{1}^{\text {nv }}(A)$ to the corresponding class of $\mathcal{L}_{\phi}$ in $\check{H}^{2}(X ; \mathbb{Z})$. By Proposition 5.6 we have $\theta^{-1}(0)=\mathrm{TM}^{\mathrm{nv}}(A)$. As a direct consequence of this observation we have: 
Corollary 5.11. Let $A$ be a homogeneous $C^{*}$-algebra such that $X$ is paracompact. If $\check{H}^{2}(X ; \mathbb{Z})=0$ then $\operatorname{IB}_{1}^{\mathrm{nv}}(A)=\mathrm{TM}^{\mathrm{nv}}(A)$.

We will now give some sufficient conditions on a trivial homogeneous $C^{*}$-algebra $A$ that will ensure the surjectivity of the map $\theta$. To do this, first recall that a topological space $X$ is said to have the Lebesgue covering dimension $d<\infty$ if $d$ is the smallest non-negative integer with the property that each finite open cover of $X$ has a refinement in which no point of $X$ is included in more than $d+1$ elements (see e.g. 9]). In this case we write $d=\operatorname{dim} X$.

Proposition 5.12. Let $X$ be a compact Hausdorff space with $\operatorname{dim} X \leq d<\infty$. For $n \geq 1$ let $A_{n}=C\left(X, \mathbb{M}_{n}\right)$. If $p=[\sqrt{(d+1) / 2}]$ then for any $n \geq p$ the mapping $\theta$ from (5.1) is surjective. In particular, if $\check{H}^{2}(X ; \mathbb{Z}) \neq 0$, then $\operatorname{TM}^{\mathrm{nv}}\left(A_{n}\right) \varsubsetneqq$ $\mathrm{IB}_{1}^{\mathrm{nv}}\left(A_{n}\right)$ for all $n \geq p$.

To prove this will use the following fact (which may be known):

Lemma 5.13. Let $X$ be a $C W$-complex with $\operatorname{dim} X=d$. Then each complex line bundle $\mathcal{L}$ over $X$ is isomorphic to a line subbundle of $X \times \mathbb{C}^{m}$ with $m=\lceil(d+1) / 2\rceil$.

Proof. We consider $\mathbb{C} P^{\infty}$ as a CW-complex in the usual way (see [16, Example 0.6]). Let $\Psi: X \rightarrow \mathbb{C} P^{\infty}$ be the classifying map of the bundle $\mathcal{L}$ (Remark [5.10). Using the cellular approximation theorem [16, Theorem 4.8] and Remark [5.10 we may assume that the map $\Psi$ is cellular, so that $\Psi$ takes the $k$-skeleton of $X$ to the $k$-skeleton of $\mathbb{C} P^{\infty}$ for all $k$. Since $\mathbb{C} P^{\infty}$ has one cell in each even dimension, $\Psi(X)$ is contained in the $d$-skeleton of $\mathbb{C} P^{\infty}$, which is the $(d-1)$-skeleton if $d$ is odd, and is $\mathbb{C} P^{m-1}$.

Hence $\mathcal{L}$ is isomorphic to the pullback $\Psi^{*}(\gamma)$ of the canonical line bundle $\gamma$ on $\mathbb{C} P^{m-1}$ (Remark 5.10), a subbundle of the trivial bundle $\mathbb{C} P^{m-1} \times \mathbb{C}^{m}$.

Proof of Proposition 5.12. Let $\mathcal{L}$ be any complex line bundle over $X$. By the proof of [32, Lemma 2.3] there exists a finite complex $Y$ with $\operatorname{dim} Y \leq d$, a continuous function $f: X \rightarrow Y$, and a line bundle $\mathcal{L}^{\prime}$ over $Y$ such that $\mathcal{L} \cong f^{*}\left(\mathcal{L}^{\prime}\right)$. By Lemma 5.13 we conclude that $\mathcal{L}^{\prime}$ is isomorphic to a line subbundle of $Y \times \mathbb{C}^{m}$, with $m=\lceil(d+1) / 2\rceil$. Hence, $\mathcal{L}$ is isomorphic to a line subbundle of $X \times \mathbb{C}^{m}$. By Remark 5.9 (b) if $n \geq p=\lceil\sqrt{m}=\lceil\sqrt{(d+1) / 2}\rceil(\lceil\sqrt{\lceil x\rceil\rceil}=\lceil\sqrt{x}$ for all $x \geq 0)$, we can assume that $\mathcal{L}$ is already a subbundle of $X \times \mathbb{M}_{n}$. By the proof of Proposition 5.8 we can find an operator $\phi \in \operatorname{IB}_{1}^{\mathrm{nv}}\left(A_{n}\right)$ such that $\mathcal{L}_{\phi}=\mathcal{L}$. By Remark 5.10 we conclude that the map $\theta$ is surjective. That $\operatorname{TM}^{\mathrm{nv}}(A) \subsetneq \mathrm{IB}_{1}^{\mathrm{nv}}\left(A_{n}\right)(n \geq p)$ when $H^{2}(X ; \mathbb{Z}) \neq 0$ follows directly from previous observations and Proposition 5.6 .

Example 5.14. Note that if $X$ is either the 2-sphere, the 2-torus or the Klein bottle, then it is well-known that $\breve{H}^{2}(X ; \mathbb{Z}) \neq 0$. In particular, if $A=C\left(X, \mathbb{M}_{n}\right)$ $(n \geq 2)$ then Proposition 5.12 shows that $\mathrm{TM}^{\mathrm{nv}}(A) \subsetneq \operatorname{IB}_{1}^{\mathrm{nv}}(A)$.

Theorem 5.15. Let $A=\Gamma_{0}(\mathcal{E})$ be a homogeneous $C^{*}$-algebra. Consider the following conditions:

(a) For every open subset $U \subset X$, each complex line subbundle of $\left.\mathcal{E}\right|_{U}$ is trivial.

(b) $\operatorname{IB}_{1}(A)=\operatorname{TM}(A)$.

(c) $\operatorname{IB}_{0,1}(A)=\mathrm{TM}_{0}(A)$.

Then $(a) \Rightarrow(b) \Rightarrow(c)$. If $A$ is separable, conditions (a), (b) and (c) are equivalent. 
Proof. (a) $\Rightarrow\left(\right.$ b): Assume (a) holds and $\phi \in \mathrm{IB}_{1}(A)$. Let $U=\operatorname{coz}(\phi)$ (open by Corollary 4.4). By Proposition 5.6 we may assume that $U \neq X$. Let $B=\Gamma_{0}\left(\left.\mathcal{E}\right|_{U}\right)$ and let $\left.\phi\right|_{U}$ be the restriction of $\phi$ to $B$. Then $\left.\phi\right|_{U} \in \operatorname{IB}_{0,1}^{\text {nv }}(B)$. By (a), $\mathcal{L}_{\phi}$ is trivial (on $U$ ) and by Proposition 5.6 we have $\left.\phi\right|_{U} \in \operatorname{TM}(B)$, that is $\left.\phi\right|_{U}=M_{c, d}$ for some $c, d \in M(B)=\Gamma_{b}\left(\left.\mathcal{E}\right|_{U}\right)$. By Remark 5.4 we can suppose that $\|c(t)\|^{2}=$ $\|d(t)\|^{2}=\left\|\phi_{t}\right\|$ for $t \in U$, so that $c, d \in B$. We can then define $a, b \in A$ by $a(t)=b(t)=0$ for $t \in X \backslash U$ and, for $t \in U, a(t)=c(t), b(t)=d(t)$. Then we have $\phi=M_{a, b} \in \operatorname{TM}_{0}(A) \subseteq \operatorname{TM}(A)$.

(b) $\Rightarrow$ (c): Take intersections with $\operatorname{IB}_{0}(A)$.

Now assume that $A$ is separable, so that $X$ is second-countable.

(c) $\Rightarrow$ (b): If $\phi \in \operatorname{IB}_{1}(A)$, take a strictly positive function $f \in C_{0}(X)$ and define $\psi \in \operatorname{IB}_{0,1}(A)$ by $\psi_{t}=f(t)^{2} \phi_{t}$. By (c) and Remark 5.4 we have $\psi=M_{c, d}$ for $c, d \in A$ with $\|c(t)\|^{2}=\|d(t)\|^{2}=\left\|\psi_{t}\right\|$. We can define $a, b \in M(A)=\Gamma_{b}(\mathcal{E})$ by $a(t)=c(t) / f(t)$ and $b(t)=d(t) / f(t)$ to get $\phi=M_{a, b} \in \mathrm{TM}(A)$.

(b) $\Rightarrow$ (a): Assume (b) holds. Let $U$ be an open subset of $X$ and $\mathcal{L}$ a complex line subbundle of $\left.\mathcal{E}\right|_{U}$. By Proposition 5.8 applied to $B=\Gamma_{0}\left(\left.\mathcal{E}\right|_{U}\right)(U$ is $\sigma$-compact since $X$ is second-countable), there is $\psi \in \mathrm{IB}_{0}(B)$ with $\mathcal{L}_{\psi}=\mathcal{L}$. Since $\left(t \mapsto\left\|\psi_{t}\right\|\right) \in$ $C_{0}(U)$, we can define $\phi \in \operatorname{IB}_{0}(A)$ by $\phi_{t}=\psi_{t}$ for $t \in U$ and $\phi_{t}=0$ for $t \in X \backslash U$. By (b), $\phi=M_{a, b}$ for $a, b \in M(A)=\Gamma_{b}(\mathcal{E})$ and then $\left.a\right|_{U}$ defines a nowhere vanishing section of $\mathcal{L}$.

Corollary 5.16. Let $A=\Gamma_{0}(\mathcal{E})$ be an $n$-homogeneous $C^{*}$-algebra with $n \geq 2$.

(a) If $X$ is second-countable with $\operatorname{dim} X<2$, or if $X$ is (homeomorphic to) a subset of a non-compact connected 2-manifold, then

$$
\operatorname{IB}_{0,1}(A)=\mathrm{TM}_{0}(A) \text { and } \operatorname{IB}_{1}(A)=\mathrm{TM}(A) .
$$

(b) If $X$ is $\sigma$-compact and contains a nonempty open subset homeomorphic to (an open subset of) $\mathbb{R}^{d}$ for some $d \geq 3$, then

$$
\mathrm{IB}_{0,1}(A) \backslash \mathrm{TM}_{0}(A) \neq \emptyset \text { and } \mathrm{IB}_{1}(A) \backslash \mathrm{TM}(A) \neq \emptyset .
$$

Remark 5.17. By a $d$-manifold we always mean a second-countable topological manifold of dimension $d$.

To prove this we will use the following facts (which are well-known to topologists).

Remark 5.18. If $X$ is a metrizable space with $\operatorname{dim} X=d<\infty$, then any locally trivial fibre bundle over $X$ can be trivialized over some open cover of $X$ consisting of at most $d+1$ elements. This follows from Dowker's and Ostrand's theorems [9, Theorems 3.2.1 and 3.2.4].

Lemma 5.19. Let $Y$ be a metrizable space with $\operatorname{dim} Y=d<\infty$ and let $X$ be a closed subset of $Y$. Then any map $f: X \rightarrow \mathbb{C} P^{\infty}$ can be, up to homotopy, continuously extended to some open neighbourhood of $X$ in $Y$.

Proof. Let $\mathcal{L}$ be a complex line bundle over $X$ defined by $f$ (Remark [5.10). By 9 , Theorem 3.1.4], we have $\operatorname{dim} X \leq \operatorname{dim} Y=d$. By Remark $5.18 \mathcal{L}$ can be trivialized over some open cover of $X$ consisting of (at most) $d+1$ elements. In particular, $\mathcal{L}$ is determined by some map $g: X \rightarrow \mathbb{C} P^{d}$ (see e.g. [18, $\left.\S 3.5\right]$ ) and by Remark $5.10 \mathrm{~g}$ is homotopic to $f$. By [17, Theorem V.7.1], finite dimensional manifolds (in particular $\mathbb{C} P^{d}$ ) are ANR spaces and so by [17, Theorem III.3.2], $g$ extends (continuously) to some open neighbourhood of $X$ in $Y$. 
Proposition 5.20. Suppose that $X$ is a locally compact subset of a non-compact connected 2-manifold $M$. Then $\check{H}^{2}(X ; \mathbb{Z})=0$.

Proof. First assume that $X=M$. Then by [28, Theorem 2.2], since every 2manifold admits a smooth structure (a classical result for which we have failed to find a complete modern reference), $X$ is homotopy equivalent to a $\mathrm{CW}$-complex of dimension $d<2$. Using Lemma 5.13 (and Remark 5.10) we conclude that $\check{H}^{2}(X ; \mathbb{Z})=0$.

Now let $X$ be an open subset of $M$. Since the previous argument applies to each connected component of $X$, we again have $\check{H}^{2}(X ; \mathbb{Z})=0$.

If $X$ is a locally compact subset of $M$, then $X$ is open in its closure $\bar{X}$. Let $Y=\bar{X} \backslash X$. Then $N=M \backslash Y$ is open in $M$ and $X$ is closed in $N$. Suppose that $\check{H}^{2}(X ; \mathbb{Z}) \neq 0$ and let $f: X \rightarrow \mathbb{C} P^{\infty}$ be any non-null-homotopic map (Remark 5.10). By Lemma 5.19 $f$ extends, up to homotopy, to a map defined on some open neighbourhood $U$ of $X$ in $M$. In particular, $\check{H}^{2}(U ; \mathbb{Z}) \neq 0$ which contradicts the second part of the proof.

Proof of Corollary 5.16, For (a) it suffices to show that $\check{H}^{2}(U ; \mathbb{Z})=0$ for all open subsets $U$ of $X$ (by Theorem 5.15 and Remark 5.10).

By Proposition 5.20 this is true if $X$ is a subset of a non-compact connected 2-manifold. Suppose that $X$ is second-countable with $\operatorname{dim} X<2$. Then for each open subset $U$ of $X$ we have $\operatorname{dim} U \leq \operatorname{dim} X$ (by the 'subset theorem' 9, Theorem 3.1.19]), so $\check{H}^{2}(U ; \mathbb{Z})=0$ (see e.g. 9, p. 94-95]).

For (b) we first choose an open subset $U \subset X$ for which $\left.\mathcal{E}\right|_{U} \cong U \times \mathbb{M}_{n}$ and such that $U$ can be considered as an open set in $\mathbb{R}^{d}(d \geq 3)$. We use the simple fact that $U$ contains an open subset that has the homotopy type of the 2 -sphere $\mathbb{S}^{2}$. So, replacing $U$ by such a subset, we can find a non-trivial line subbundle $\mathcal{L}$ of $U \times \mathbb{C}^{2}$. By Remark 5.9 (b) we may assume that $\mathcal{L}$ is a subbundle of $U \times\left.\mathbb{M}_{n} \cong \mathcal{E}\right|_{U}$. The assertion now follows from the proof of Theorem 5.15.

Remark 5.21. In the literature there are somewhat similar phonomena that arise for unital $C^{*}$-algebras $A$ of sections of a $C^{*}$-bundle over a (second-countable) compact Hausdorff space $X$. The question was to describe when the set $\operatorname{Aut}_{C(X)}(A)$ of all $C(X)$-linear automorphisms of such $A$ coincides with the inner automorphisms of $A$ (see e.g. [22, 34, 30, 31]). For example, if $A$ is any separable unital continuous trace $C^{*}$-algebra with (primitive) spectrum $X$, there always exists an exact sequence

$$
0 \longrightarrow \operatorname{InnAut}(A) \longrightarrow \operatorname{Aut}_{C(X)}(A) \stackrel{\eta}{\longrightarrow} \check{H}^{2}(X ; \mathbb{Z})
$$

of abelian groups. In general, $\eta$ does not need to be surjective unless $A$ is stable [30, Theorem 2.1]. If $A$ is $n$-homogeneous then the image of $\eta$ is contained in the torsion subgroup of $\check{H}^{2}(X ; \mathbb{Z})[30,2.19]$. In particular, $A=C\left(\mathbb{S}^{2}, \mathbb{M}_{2}\right)$ shows that it can happen that $\operatorname{TM}^{\mathrm{nv}}(A) \subsetneq \mathrm{IB}_{1}^{\mathrm{nv}}(A)$ even though $\operatorname{Aut}_{C\left(\mathbb{S}^{2}\right)}(A)=\operatorname{InnAut}(A)$ (since $\check{H}^{2}\left(\mathbb{S}^{2} ; \mathbb{Z}\right) \cong \mathbb{Z}$ is torsion free). Our Proposition 5.12 shows that the map $\theta$ from (5.1) is surjective in this case. In contrast to $\eta$, there is no obvious group structure on the domain $\operatorname{IB}_{1}^{\mathrm{nv}}(A)$ of $\theta$.

\section{Closure of $\operatorname{TM}(A)$ on homogeneous $C^{*}$-algebras}

Here we continue to work with $n$-homogeneous algebras $A=\Gamma_{0}(\mathcal{E})$. The class $\mathrm{IB}_{1}(A)$ considered in $\$ 5$ is rather obviously designed to capture a restriction on the 
closure of $\operatorname{TM}(A)$ (and similarly $\operatorname{IB}_{0,1}(A)$ should relate to the closure of $\mathrm{TM}_{0}(A)$ ). We verify right away (Proposition 6.1) that $\mathrm{IB}_{1}(A)$ and $\mathrm{IB}_{0,1}(A)$ are indeed closed. However, further restrictions on the operators $\phi$ in the closure of $\operatorname{TM}(A)$ arise because triviality of the line bundles $\mathcal{L}_{\psi}$ associated with $\psi \in \mathrm{TM}(A)$ is still present for the line bundle $\mathcal{L}_{\phi}$ provided $U=\operatorname{coz}(\phi)$ is compact (see Corollary 6.5). If $U$ is not compact, this triviality is evident on compact subsets of $U$ (see Theorem 6.9. where we characterize the closure of $\mathrm{TM}_{0}(A)$ ). However $\mathcal{L}_{\phi}$ need not be trivial globally on $U$ (so that $\phi \notin \mathrm{TM}(A)$ is possible) and this led us to define the concept of a phantom bundle (Definition 6.11). The terminology is by analogy with the well known concept of a phantom map (see [27). Thus, in Corollary 6.12, we see that finding $\phi$ in the norm closure of $\operatorname{TM}_{0}(A)$ with $\phi \notin \mathrm{TM}_{0}(A)$ is directly related to finding suitable phantom complex line bundles.

For these to exist, we need $U$ to have a rather complicated algebraic topological structure, and we find examples with $\pi_{1}(U) \cong \mathbb{Q}$ (Proposition 6.17). In fact, we can also find such examples when $X$ contains (a copy of) an open subset of $\mathbb{R}^{d}$ with $d \geq 3$ and $n \geq 2$ (Theorem 6.18).

Proposition 6.1. Let $A$ be a homogeneous $C^{*}$-algebra. Then $\operatorname{IB}_{1}(A)$ and $\operatorname{IB}_{0,1}(A)$ are norm closed subsets of $\mathcal{B}(A)$.

Proof. If $\left(\phi_{k}\right)_{k=1}^{\infty}$ is a sequence in $\operatorname{IB}_{1}(A)$ that converges in operator norm to $\phi \in$ $\mathcal{B}(A)$, then it is clear that $\phi(I) \subset I$ for each ideal $I$ of $A$. Thus $\phi \in \operatorname{IB}(A)$.

By (2.2) we have $\left\|\phi-\phi_{k}\right\|=\sup _{t \in X}\left\|\phi_{t}-\left(\phi_{k}\right)_{t}\right\|$ and so $\lim _{k \rightarrow \infty}\left(\phi_{k}\right)_{t}=\phi_{t} \in$ $\mathcal{B}\left(A_{t}\right)$ (for $\left.t \in X\right)$. Since $A_{t} \cong \mathbb{M}_{n}$, invoking Theorem 3.4. we have $\phi_{t} \in \operatorname{TM}\left(A_{t}\right)$ (for $t \in X$ ) and hence $\phi \in \operatorname{IB}_{1}(A)$.

If $\phi_{k} \in \mathrm{IB}_{0,1}(A)$ for each $k$, then $\left\|\left(\phi_{k}\right)_{t}\right\| \rightarrow\left\|\phi_{t}\right\|$ uniformly for $t \in X$. As $\left(t \mapsto\left\|\left(\phi_{k}\right)_{t}\right\|\right) \in C_{0}(X)$, it follows that $\left(t \mapsto\left\|\phi_{t}\right\|\right) \in C_{0}(X)$ and so $\phi \in \operatorname{IB}_{0}(A)$.

Lemma 6.2. Let $A$ be a homogeneous $C^{*}$-algebra, and let $\phi \in \operatorname{IB}_{1}^{\mathrm{nv}}(A)$. Then there is $\psi \in \operatorname{IB}_{1}^{\mathrm{nv}}(A)$ with $\psi_{t}=\phi_{t} /\left\|\phi_{t}\right\|$ for each $t \in X$.

Moreover $\phi \in \mathrm{TM}(A) \Longleftrightarrow \psi \in \operatorname{TM}(A)$.

Proof. Since $t \mapsto\left\|\phi_{t}\right\|$ is continuous by Corollary 4.4 we can define $\psi_{t}=\phi_{t} /\left\|\phi_{t}\right\|$ and get $\psi \in \mathrm{IB}(A)$ via local applications of Proposition 4.2. Clearly $\psi \in \mathrm{IB}_{1}^{\mathrm{nv}}(A)$.

If $\phi=M_{a, b} \in \operatorname{TM}(A)$ for $a, b \in M(A)=\Gamma_{b}(\mathcal{E})$, then we can normalize $a$ and $b$ as in Remark 5.4 and then take $c, d \in A$ with $c(t)=a(t) / \sqrt{\left\|\phi_{t}\right\|}, d(t)=b(t) / \sqrt{\left\|\phi_{t}\right\|}$ to get $\psi=M_{c, d}$. So $\psi \in \operatorname{TM}(A)$. We can reverse this argument.

Remark 6.3. Let $\overline{\overline{\mathrm{TM}(A)}}$ denote the operator norm closure of $\operatorname{TM}(A)$, and similarly for $\overline{\overline{\mathrm{TM}_{0}(A)}}$. If $A$ is homogeneous, then Proposition 6.1 gives $\overline{\overline{\mathrm{TM}(A)}} \subset \operatorname{IB}_{1}(A)$ and $\overline{\overline{\operatorname{TM}_{0}(A)}} \subset \operatorname{IB}_{0,1}(A)$.

Proposition 6.4. Let $A=\Gamma_{0}(\mathcal{E})$ be an n-homogeneous $C^{*}$-algebra. Suppose that $\phi \in \overline{\overline{\operatorname{TM}(A)}}$ such that $\inf _{t \in X}\left\|\phi_{t}\right\|=\delta>0$. Then $\phi \in \operatorname{TM}(A)$.

Proof. Let $\left(\phi_{k}\right)_{k=1}^{\infty}$ be a sequence in $\operatorname{TM}(A)$ with $\lim _{k \rightarrow \infty} \phi_{k}=\phi \in \mathcal{B}(A)$. For $k$ large enough that $\left\|\phi_{k}-\phi\right\|<\delta / 2$ we must have $\left\|\left(\phi_{k}\right)_{t}\right\|>\delta / 2$ for each $t \in X$ (and hence $\left.\phi_{k} \in \mathrm{IB}_{1}^{\mathrm{nv}}(A)\right)$. With no loss of generality we may assume that this holds for all $k \geq 1$.

Since

$$
\sup _{t \in X}\left|\left\|\left(\phi_{k}\right)_{t}\right\|-\left\|\phi_{t}\right\|\right| \leq \sup _{t \in X}\left\|\left(\phi_{k}\right)_{t}-\phi_{t}\right\|=\left\|\phi_{k}-\phi\right\|,
$$


we may use Lemma 6.2 to normalise each $\phi_{k}$ and $\phi$ and assume that

$$
1=\|\phi\|=\left\|\phi_{t}\right\|=\left\|\left(\phi_{k}\right)_{t}\right\|=\left\|\phi_{k}\right\|
$$

holds for all $k \geq 1$ and $t \in X$ (and still $\lim _{k \rightarrow \infty} \phi_{k}=\phi$ ).

We now write $\phi_{k}=M_{a_{k}, b_{k}}$ for $a_{k}, b_{k} \in M(A)=\Gamma_{b}(\mathcal{E})$ such that $\left\|a_{k}(t)\right\|=$ $\left\|b_{k}(t)\right\|=1$ (for all $t \in X$ and all $k$ ). We consider the line bundle $\mathcal{L}_{\phi}$ associated with $\phi$ according to Proposition 5.6 which is locally expressible as $\{(t, \lambda a(t))\}$, where $\phi_{t}=M_{a(t), b(t)}$ locally. We assume, as we can, that $\|a(t)\|=\|b(t)\|=1$ (locally).

Let $0<\varepsilon<(18 n)^{-1 / 2}$.

By Remark 4.1 (d), for $k$ suitably large (but fixed) and $t \in X$ arbitrary, we have $\left\|\left(\phi_{k}\right)_{t}-\phi_{t}\right\|_{c b}<\varepsilon$. Since, by Mathieu's theorem (Theorem 2.1), we locally have

$$
\left\|\left(\phi_{k}\right)_{t}-\phi_{t}\right\|_{c b}=\left\|M_{a_{k}(t), b_{k}(t)}-M_{a(t), b(t)}\right\|_{c b}=\left\|a_{k}(t) \otimes b_{k}(t)-a(t) \otimes b(t)\right\|_{h},
$$

by Lemma 3.1 we can locally find a scalar $\mu_{k}(t)$ of modulus 1 such that

$$
\left\|a_{k}(t)-\mu_{k}(t) a(t)\right\|<6 \varepsilon
$$

(note that $(18 n)^{-1 / 2}<1 / 3$ for all $n \geq 1$ ).

Consider the inner product $\langle\cdot, \cdot\rangle_{2}$ defined in Remark 4.1 (g). We claim that locally $\left\langle a_{k}(t), a(t)\right\rangle_{2} \neq 0$. Indeed, first note that (locally)

$$
\left|\left\langle a_{k}(t), a(t)\right\rangle_{2}\right|=\left|\left\langle a_{k}(t), \mu_{k}(t) a(t)\right\rangle_{2}\right|
$$

and by (4.2)

$$
\left\|a_{k}(t)\right\|_{2} \geq 1, \quad\left\|\mu_{k}(t) a(t)\right\|_{2} \geq 1, \quad\left\|a_{k}(t)-\mu_{k}(t) a(t)\right\|_{2}<6 \sqrt{n} \varepsilon .
$$

Since any two vectors $v$ and $w$ of norm at least 1 in a Hilbert space satisfy

$$
\|v-w\|_{2}^{2} \geq\|v\|_{2}^{2}+\|w\|_{2}^{2}-2\left|\langle v, w\rangle_{2}\right| \geq 2\left(1-\left|\langle v, w\rangle_{2}\right|\right),
$$

letting $v=a_{k}(t)$ and $w=\mu_{k}(t) a(t)$, we have (locally)

$$
\begin{aligned}
\left|\left\langle a_{k}(t), \mu_{k}(t) a(t)\right\rangle_{2}\right| & \geq 1-\frac{1}{2}\left\|a_{k}(t)-\mu_{k}(t) a(t)\right\|_{2}^{2}>1-18 n \varepsilon^{2} \\
& >0 .
\end{aligned}
$$

We can therefore define $a^{\prime}(t)$ locally as the normalised (in operator norm) orthogonal projection

$$
a^{\prime}(t)=\frac{\left\langle a_{k}(t), a(t)\right\rangle_{2}}{\left|\left\langle a_{k}(t), a(t)\right\rangle_{2}\right|} \cdot a(t) .
$$

Then $t \mapsto a^{\prime}(t)$ is locally well-defined and continuous (by Remark $4.1(\mathrm{~g})$ ).

As $a^{\prime}(t)$ is independent of multiplying $a(t)$ by unit scalars, it defines a nowhere vanishing global section of $\mathcal{L}_{\phi}$. By Proposition 5.6, we must have $\phi \in \operatorname{TM}(A)$, as required.

Corollary 6.5. Let $A$ be a unital homogeneous $C^{*}$-algebra. Then

$$
\overline{\overline{\mathrm{TM}(A)}} \cap \operatorname{IB}_{1}^{\mathrm{nv}}(A) \subset \mathrm{TM}(A) .
$$

Proof. Let $\phi \in \overline{\overline{\operatorname{TM}(A)}} \cap \operatorname{IB}_{1}^{\text {nv }}(A)$. Since $t \mapsto\left\|\phi_{t}\right\|$ is continuous (Corollary 4.4 ) and never vanishing on $X$ (which is compact, as $A$ is unital), it has a minimum value $\delta>0$. By Proposition 6.4 $\phi \in \operatorname{TM}(A)$. 
Example 6.6. Let $A=C\left(X, \mathbb{M}_{n}\right)(n \geq 2)$, where $X$ is any compact Hausdorff space with $\operatorname{dim} X \leq 7$ and $\check{H}^{2}(X ; \mathbb{Z}) \neq 0$. Then $\overline{\overline{\operatorname{TM}(A)}} \subsetneq \operatorname{IB}_{1}(A)$. Indeed, by Proposition 5.12 there exists $\phi \in \operatorname{IB}_{1}^{\mathrm{nv}}(A) \backslash \mathrm{TM}(A)$. By Corollary 6.5, $\phi \notin \overline{\overline{\operatorname{TM}(A)}}$. (Since $A$ is unital, $\operatorname{TM}_{0}(A)=\operatorname{TM}(A)$ and $\operatorname{IB}_{0,1}(A)=\operatorname{IB}_{1}(A)$.)

Corollary 6.7. If $A=\Gamma_{0}(\mathcal{E})$ is a homogeneous $C^{*}$-algebra, then both $\operatorname{Inn} \operatorname{Aut}_{\text {alg }}(A)$ and $\operatorname{InnAut}(A)$ (see (2.3)) are norm closed.

Proof. If $M_{a, a^{-1}} \in \operatorname{InnAut}_{\text {alg }}(A)$, then for all $t \in X$ we have $\left\|\left(M_{a, a^{-1}}\right)_{t}\right\|=$ $\|a(t)\|\left\|a(t)^{-1}\right\| \geq 1$. Hence if $\phi$ is in the norm closure of $\operatorname{InnAutalg}_{\operatorname{lal}}(A)$, we have $\left\|\phi_{t}\right\| \geq 1$ for each $t \in X$. By Proposition 6.4, $\phi=M_{b, c}$ for some $b, c \in M(A)$. Since $\phi_{t}(1)=1, c(t)=b(t)^{-1}$ for each $t$ and so $c=b^{-1} \in M(A)=\Gamma_{b}(\mathcal{E})$.

The proof for the $\operatorname{InnAut}(A)$ is similar.

Remark 6.8. The results that $\operatorname{Inn} A u t(A)$ is norm closed if the $C^{*}$-algebra $A$ is prime or homogeneous (in Corollaries 3.5 and 6.7) can also be deduced from 35, 19, 3. To explain the deductions, we first identify $\operatorname{Inn} A u t(A)$ with $\operatorname{Inn} A u t(M(A))$.

If $A$ is prime, then $M(A)$ is also prime (by [2, Lemma 1.1.7]). In particular, $\operatorname{Orc}(M(A))=1$ (in the sense of [35, §2]), so by [35, Corollary 4.6] inner derivations of $M(A)$ are norm closed. Then [19, Theorem 5.3] implies that $\operatorname{InnAut}(M(A))$ is also norm closed.

If $A$ is homogeneous (or more generally quasi-central and quasi-standard in the sense of [3]), then $M(A)$ is quasi-standard [3] Corollary 4.10]. Thus we have $\operatorname{Orc}(M(A))=1$, and we may conclude as in the prime case.

Theorem 6.9. Let $A=\Gamma_{0}(\mathcal{E})$ be a homogeneous $C^{*}$-algebra. For an operator $\phi \in \mathcal{B}(A)$, the following two conditions are equivalent:

(a) $\phi \in \overline{\overline{\operatorname{TM}_{0}(A)}}$.

(b) $\phi \in \operatorname{IB}_{0,1}(A)$ and for $U=\operatorname{coz}(\phi)$ (open by Corollary 4.4) $\mathcal{L}_{\phi}$ is trivial on each compact subset of $U$.

Proof. (a) $\Rightarrow$ (b): Let $\phi \in \overline{\overline{\operatorname{TM}_{0}(A)}}$, so that $\phi \in \operatorname{IB}_{0,1}(A)$ (Remark 6.3). For each compact subset $K \subset U$, we have $\phi_{K} \in \overline{\overline{\operatorname{TM}\left(A_{K}\right)}}$ (recall that $A_{K}=\Gamma\left(\left.\mathcal{E}\right|_{K}\right)$ by Remark 4.1 (b)). By Corollary 6.5 we have $\phi_{K} \in \mathrm{TM}\left(A_{K}\right)$, so that $\mathcal{L}_{\phi}$ must be trivial on $K$ (by Proposition 5.6).

(b) $\Rightarrow$ (a): Let $\phi \in \operatorname{IB}_{0,1}(A)$, so that $t \mapsto\left\|\phi_{t}\right\|$ is in $C_{0}(X)$.

For any sequence $\delta_{n}>0$ decreasing strictly to 0 (for instance $\delta_{n}=1 / n$ ) let

$$
K_{n}=\left\{t \in X:\left\|\phi_{t}\right\| \geq \delta_{n}\right\} .
$$

Then each $K_{n}$ is compact, $K_{n} \subset K_{n+1}^{\circ}$ and $\bigcup_{n=1}^{\infty} K_{n}=U$. By Proposition 5.6. $\psi_{K_{n}} \in \operatorname{TM}\left(A_{K_{n}}\right)=\operatorname{TM}\left(\Gamma\left(\left.\mathcal{E}\right|_{K_{n}}\right)\right)$ and so there are $a_{n}, b_{n} \in A_{K_{n}}$ with $\psi_{K_{n}}=$ $M_{a_{n}, b_{n}}$. Using Remark 5.4 we may assume $\left\|a_{n}(t)\right\|=\left\|b_{n}(t)\right\|=\sqrt{\left\|\phi_{t}\right\|}$ for $t \in K_{n}$. By Remark 4.1 (b) we may extend $a_{n}$ to $c_{n} \in A$ with $c_{n}(t)=0$ for $t \in X \backslash K_{n+1}^{\circ}$ and $\left\|c_{n}(t)\right\|^{2} \leq \delta_{n}$ for all $t \in X \backslash K_{n}$. Similarly we extend $b_{n}$ to $d_{n} \in A$ supported in $K_{n+1}^{\circ}$ with $\left\|d_{n}(t)\right\|^{2} \leq \delta_{n}$ for $t \in X \backslash K_{n}$. Then $\left(M_{c_{n}, d_{n}}-\phi\right)_{t}$ has norm at most $2 \delta_{n}$ for all $t \in X$ and hence $\lim _{n \rightarrow \infty} M_{c_{n}, d_{n}}=\phi$. Thus $\phi \in \overline{\overline{\operatorname{TM}_{0}(A)}}$.

Corollary 6.10. For a homogeneous $C^{*}$-algebra $A=\Gamma_{0}(\mathcal{E})$ the following conditions are equivalent:

(a) $\overline{\overline{\operatorname{TM}_{0}(A)}}=\operatorname{IB}_{0,1}(A)$. 
(b) For each $\sigma$-compact open subset $U$ of $X$, every complex line subbundle of $\left.\mathcal{E}\right|_{U}$ is trivial on all compact subsets of $U$.

Proof. $(\mathrm{a}) \Rightarrow(\mathrm{b})$ : Let $U$ be a $\sigma$-compact open subset of $X, B=\Gamma_{0}\left(\left.\mathcal{E}\right|_{U}\right)$ and $\mathcal{L}$ a complex line subbundle of $\left.\mathcal{E}\right|_{U}$. By Proposition 5.8 we can find an operator $\phi \in \operatorname{IB}_{0,1}^{\text {nv }}(B)$ such that $\mathcal{L}_{\phi}=\mathcal{L}$. By extending $\phi$ to be zero outside $U$, we may assume that $\phi \in \mathrm{IB}_{0,1}(A)$, so that $U=\operatorname{coz}(\phi)$. By assumption, $\phi \in \overline{\overline{\operatorname{TM}_{0}(A)}}$, so by Theorem 6.9 $\mathcal{L}$ is trivial on all compact subsets of $U$.

(b) $\Rightarrow(\mathrm{a})$ : If $\phi \in \mathrm{IB}_{0,1}(A)$ then $U=\operatorname{coz}(\phi)$ is an open, necessarily $\sigma$-compact subset of $X$ (since $t \mapsto\left\|\phi_{t}\right\|$ is in $C_{0}(X)$ ). By assumption, $\mathcal{L}_{\phi}$ is trivial on every compact subset of $U$. Hence, $\phi \in \overline{\overline{\mathrm{TM}_{0}(A)}}$ by Theorem 6.9 .

Definition 6.11. A locally trivial fibre bundle $\mathcal{F}$ over a locally compact Hausdorff space $X$ is said to be a phantom bundle if $\mathcal{F}$ is not globally trivial, but is trivial on each compact subset of $X$.

Corollary 6.12. Let $A=\Gamma_{0}(\mathcal{E})$ be a homogeneous $C^{*}$-algebra. Then $\operatorname{TM}_{0}(A)$ fails to be norm closed in $\mathcal{B}(A)$ if and only if there exists a $\sigma$-compact open subset $U$ of $X$ and a phantom complex line subbundle of $\left.\mathcal{E}\right|_{U}$.

If these equivalent conditions hold, then $\mathrm{TM}(A)$ fails to be norm closed.

Proof. If $\mathrm{TM}_{0}(A)$ fails to be norm closed, there is $\phi \in \overline{\overline{\operatorname{TM}_{0}(A)}} \backslash \operatorname{TM}_{0}(A)$. Note that $\phi \in \mathrm{IB}_{0,1}(A)$ by Proposition 6.1. By Theorem 6.9, for $U=\operatorname{coz}(\phi)$ (open and $\sigma$-compact), $\mathcal{L}_{\phi}$ is trivial on each compact subset of $U$. Moreover $\left.\phi\right|_{U} \in \operatorname{IB}_{0,1}^{\mathrm{nv}}(B)$ for $B=\Gamma_{0}\left(\left.\mathcal{E}\right|_{U}\right)$. By Proposition 5.6, if $\mathcal{L}_{\phi}$ is globally trivial, then $\left.\phi\right|_{U} \in \operatorname{TM}(B) \cap$ $\mathrm{IB}_{0}(B)=\mathrm{TM}_{0}(B)$. So $\left.\phi\right|_{U}=M_{a, b}$ for $a, b \in B$. Since $B$ can be considered as an ideal of $A$ (Remark 4.1 (c)), we treat $a, b \in A$. Hence $\phi=M_{a, b} \in \mathrm{TM}_{0}(A)$, a contradiction. Thus $\mathcal{L}_{\phi}$ is a phantom bundle.

Conversely, suppose that $U \subset X$ is open and $\sigma$-compact and that $\mathcal{L}$ is a phantom complex line subbundle of $\left.\mathcal{E}\right|_{U}$. Then, taking $B=\Gamma_{0}\left(\left.\mathcal{E}\right|_{U}\right)$, Proposition 5.8 provides $\psi \in \operatorname{IB}_{0,1}^{\text {nv }}(B)$ with $\mathcal{L}_{\psi}=\mathcal{L}$. As $\mathcal{L}$ is a phantom bundle, by Proposition [5.6] $\psi \notin \mathrm{TM}(B)$. We may define $\phi \in \operatorname{IB}_{0,1}(A)$ by $\phi_{t}=\psi_{t}$ for $t \in U$ and $\phi_{t}=0$ for $t \in X \backslash U$. From $\psi=\left.\phi\right|_{U} \notin \operatorname{TM}(B)$, we have $\phi \notin \operatorname{TM}(A)$ but $\phi \in \overline{\overline{\operatorname{TM}_{0}(A)}}$ by Theorem 6.9.

We now describe below a class of homogeneous $C^{*}$-algebras $A$ for which $\operatorname{TM}_{0}(A)$ and $\operatorname{TM}(A)$ both fail to be norm closed. We first explain some preliminaries.

Remark 6.13. Let $G$ be a group and $n$ a positive integer. Recall that a space $X$ is called an Eilenberg-MacLane space of type $K(G, n)$, if it's $n$-th homotopy group $\pi_{n}(X)$ is isomorphic to $G$ and all other homotopy groups trivial. If $n>1$ then $G$ must be abelian (since for all $n>1$, the homotopy groups $\pi_{n}(X)$ are abelian). We state some basic facts and examples about Eilenberg-MacLane spaces:

(a) There exists a CW-complex $K(G, n)$ for any group $G$ at $n=1$, and abelian group $G$ at $n>1$. Moreover such a $\mathrm{CW}$-complex is unique up to homotopy type. Hence, by abuse of notation, it is common to denote any such space by $K(G, n)$ [16, p. 365-366].

(b) Given a CW-complex $X$, there is a bijection between its cohomology group $H^{n}(X ; G)$ and the homotopy classes $[X, K(G, n)]$ of maps from $X$ to $K(G, n)$ [16, Theorem 4.57]. 
(c) $K(\mathbb{Z}, 2) \cong \mathbb{C} P^{\infty}$ [16. Example 4.50]. In particular, by (b) and Remark 5.10, for each CW-complex $X$ there is a bijection between $[X, K(\mathbb{Z}, 2)]$ and isomorphism classes of complex line bundles over $X$.

Proposition 6.14. If $X$ is a locally compact $C W$-complex of type $K(\mathbb{Q}, 1)$, then every non-trivial complex line bundle over $X$ is a phantom bundle. Moreover there are uncountably many non-isomorphic such bundles.

Proof. The standard model of $K(\mathbb{Q}, 1)$ is the mapping telescope $\Delta$ of the sequence

$$
\mathbb{S}^{1} \stackrel{f_{1}}{\longrightarrow} \mathbb{S}^{1} \stackrel{f_{2}}{\longrightarrow} \mathbb{S}^{1} \stackrel{f_{3}}{\longrightarrow} \cdots,
$$

where $f_{n}: \mathbb{S}^{1} \rightarrow \mathbb{S}^{1}$ is given by $z \mapsto z^{n+1}$ (see e.g. 7, Example 1.9] and [16, Section 3.F]).

We first consider the case when $X=\Delta$. Applying $H_{1}(-; \mathbb{Z})$ to the levels of the mapping telescope (6.1) gives the system

$$
\mathbb{Z} \stackrel{\left(f_{1}\right)_{*}}{\longrightarrow} \mathbb{Z} \stackrel{\left(f_{2}\right)_{*}}{\longrightarrow} \mathbb{Z} \stackrel{\left(f_{3}\right)_{*}}{\longrightarrow} \cdots,
$$

where $\left(f_{n}\right)_{*}: \mathbb{Z} \rightarrow \mathbb{Z}$ is given by $k \mapsto(n+1) k$ (see [16, Section 3.F]). The colimit of this system is (by [16. Proposition 3.33]) $H_{1}(\Delta ; \mathbb{Z})=\mathbb{Q}$ and all other integral homology groups are trivial. By the universal coefficient theorem for cohomology [16. Theorem 3.2] (see also [16, §3.F]) each integral cohomology group of $\Delta$ is trivial, except for $\check{H}^{2}(\Delta ; \mathbb{Z})$ which is isomorphic to $\operatorname{Ext}(\mathbb{Q} ; \mathbb{Z})$. By $[38] \operatorname{Ext}(\mathbb{Q} ; \mathbb{Z})$ is isomorphic to the additive group of real numbers. Hence, by Remark 5.10 , there exists uncountably many non-isomorphic complex line bundles over $\Delta$. We claim that each non-trivial such bundle $\mathcal{L}$ is a phantom bundle. Indeed, for $n \geq 1$ let $\Delta_{n}$ denote the $n$-the level of the mapping telescope (6.1). If $K$ be an arbitrary compact subset of $\Delta$ then $K$ is contained in some $\Delta_{n}$. Since all $\Delta_{n}$ 's are homotopy equivalent to $\mathbb{S}^{1}$, and since $\check{H}^{2}\left(\mathbb{S}^{1} ; \mathbb{Z}\right)=0$, we conclude that $\left.\mathcal{L}\right|_{\Delta_{n}}$ is trivial. Then $\left.\mathcal{L}\right|_{K}$ is also trivial, since $K \subset \Delta_{n}$.

If $X$ is another locally compact CW-complex of of type $K(\mathbb{Q}, 1)$, then by Remark 6.13 (a), there are maps $f: \Delta \rightarrow X$ and $g: X \rightarrow \Delta$ such that $g \circ f$ and $f \circ g$ are homotopic (respectively) to the identity maps (on $\Delta$ and $X$, respectively). If $\mathcal{L}$ is a non-trivial complex line bundle over $\Delta$, then $g^{*}(\mathcal{L})$ is non-trivial over $X$ (Remark 5.10). Moreover $g^{*}(\mathcal{L})$ is a phantom bundle because $K \subset X$ compact implies $g(K) \subset \Delta$ compact and $\left.g^{*}(\mathcal{L})\right|_{K}$ is a restriction of $\left.g^{*}(\mathcal{L})\right|_{g^{-1}(g(K))}=g^{*}\left(\left.\mathcal{L}\right|_{g(K)}\right)$, which is a trivial bundle. Since $g$ is a homotopy equivalence, every non-trivial complex line bundle over $X$ must be isomorphic to $g^{*}(\mathcal{L})$ for some $\mathcal{L}$.

Remark 6.15. With the same notation as in the proof of Proposition 6.14, one can show that for each compact subset $K$ of $\Delta$ we have $\breve{H}^{2}(K ; \mathbb{Z})=0$. To sketch the proof, choose an arbitrary complex line bundle $\mathcal{L}$ over $K$. Then using Lemma 5.19 (and Remark 5.10) $\mathcal{L}$ can be extended to an open neighbourhood $U$ of $K$. The assertion can now be established via an argument with triangulations of $\Delta$. There is a triangulation of $\Delta$ where $\Delta_{1}$ has 3 triangles and each $\Delta_{n+1}$ has $n+3$ more triangles than $\Delta_{n}$. We may subdivide the triangles that touch $K$ to get finitely many that cover $K$ and are all contained in $U$. Now consider the union $T$ of the triangles that touch $K$. It is enough to show $\left.\mathcal{L}\right|_{T}$ is trivial. We can deformation retract $T$ to a union of 1 -simplices. To do so, work on one triangle (2-cell) at a time, starting with any 2-cell in $\Delta_{1}$ with a 'free' edge not in the boundary of $\Delta_{1}$ relative to $\Delta_{2}$ (where 'free' means the edge does not bound a second 2-cell). After 
each step, consider the remaining 2-cells, edges and vertices. Move on to $\Delta_{2}$ once all 2-cells in $\Delta_{1}$ are exhausted, etc, so as to arrive at a 1-simplex after finitely many steps. As all complex line bundles over 1-simplices are trivial, we have that $\left.\mathcal{L}\right|_{T}$ is trivial.

In private correspondence, Mladen Bestvina informed us that we can find phantom bundles even over some open subset of $\mathbb{R}^{3}$, and referred us to [6]. We outline the construction of such a subset.

Proposition 6.16. There exists an open subset $\Omega$ of $\mathbb{R}^{3}$ of type $K(\mathbb{Q}, 1)$.

Proof. In 6, a construction is given of dense open sets $U$ in the 3 -sphere $\mathbb{S}^{3}$ with fundamental groups $\pi_{1}(U)$ that are large subgroups of $\mathbb{Q}$. Given a sequence $n_{i}$ of natural numbers $n_{i}>1, \pi_{1}(U)$ can be $\left\{p / q \in \mathbb{Q}: p \in \mathbb{Z}, q=\prod_{i=1}^{k} n_{i}\right.$ for some $\left.k\right\}$. In particular we will take $n_{i}=i+1$ and then $\pi_{1}(U)=\mathbb{Q}$.

The construction defines $U$ as a union of closed solid tori $U=\bigcup_{i=1}^{\infty} S_{i}$. For each $i$, both $S_{i}$ and the complement of its interior $T_{i}=\mathbb{S}^{3} \backslash S_{i}^{\circ}$ are solid tori with intersection $S_{i} \cap T_{i}$ a (2-dimensional) torus. At each step, $T_{i+1}$ is constructed inside $T_{i}$ as an unknotted solid torus of smaller cross-sectional area that winds $n_{i}$ times around the meridian circle of $T_{i}$. Since $T_{i+1}$ can be unfolded to a standard embedding of a torus via an ambient isotopy of $\mathbb{S}^{3}, S_{i+1}$ must be a solid torus.

Let $f: \mathbb{S}^{n} \rightarrow U$ be an arbitrary map. Then $f$ maps $\mathbb{S}^{n}$ into one of the solid tori $S_{i}$ and these are homotopic to their meridian circle. In particular $\pi_{n}(U)=0$ for all $n>1$. By Remark 6.13 $U$ has the type $K(\mathbb{Q}, 1)$.

Choose any point $t \in \mathbb{S}^{3} \backslash U$. Since $\mathbb{S}^{3} \backslash\{t\}$ is homeomorphic to $\mathbb{R}^{3}$, say via the homeomorphism $F$, then $\Omega=F(U)$ is an open subset of $\mathbb{R}^{3}$ of the type $K(\mathbb{Q}, 1)$.

Proposition 6.17. Let $X$ be any locally compact $\sigma$-compact $C W$-complex of type $K(\mathbb{Q}, 1)$ (e.g. $X=\Delta$ ). Then the $C^{*}$-algebra $A=C_{0}\left(X, \mathbb{M}_{n}\right)(n \geq 2)$ has the following property:

There exists an operator $\phi \in \operatorname{IB}_{0,1}^{\mathrm{nv}}(A) \backslash \mathrm{TM}(A)$ such that $\phi$ is in the norm closure of $\mathrm{TM}_{c p}(A) \cap \mathrm{TM}_{0}(A)=\left\{M_{a, a^{*}}: a \in A\right\}$.

In particular, $\operatorname{TM}_{0}(A), \operatorname{TM}(A)$ and $\mathrm{TM}_{c p}(A)$ all fail to be norm closed.

Further, if $X=\Delta$, we have $\overline{\overline{\operatorname{TM}_{0}(A)}}=\operatorname{IB}_{0,1}(A)$.

Proof. Choose any phantom complex line bundle $\mathcal{L}$ over $X$ (Proposition 6.14). Since, by Remark 6.13 (a), $X$ has the same homotopy type as the space $\Delta$ of Proposition 6.14 (which is a 2-dimensional complex), using Remark 5.10 and Lemma 5.13 we may assume that $\mathcal{L}$ is a subbundle of the trivial bundle $X \times \mathbb{C}^{2}$. We also realise $\mathbb{C}^{2}$ as a subset of $\mathbb{M}_{n}$ as $\left\{z_{1} e_{1,1}+z_{2} e_{1,2}: z_{1}, z_{2} \in \mathbb{C}\right\}$, and in this way consider $\mathcal{L}$ a subbundle of $X \times \mathbb{M}_{n}$. By the proof of Proposition 5.8 we can find two sections $a, b$ of $\mathcal{L}$ vanishing at infinity (so that $a, b \in A$ ) such that $\operatorname{span}\{a(t), b(t)\}=\mathcal{L}_{t}$ for each $t \in X$.

We define a map

$$
\phi: A \rightarrow A \quad \text { by } \phi=M_{a, a^{*}}+M_{b, b^{*}} .
$$

Then $\phi$ defines a completely positive elementary operator on $A$ of length at most 2 . Clearly, $\phi_{t} \neq 0$ for all $t \in X$, so $\phi \in \operatorname{IB}_{0,1}^{\text {nv }}(A)$. Also, $\mathcal{L}_{\phi}=\mathcal{L}$. Since the bundle $\mathcal{L}$ is non-trivial, by Proposition 5.6 we have $\phi \notin \mathrm{TM}(A)$. On the other hand, since $\mathcal{L}$ is

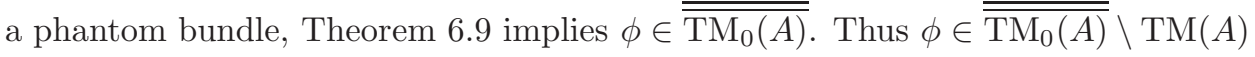
and consequently $\phi$ has length 2 . 
We have $\phi_{K}$ completely positive on $A_{K}=\Gamma\left(\left.\mathcal{E}\right|_{K}\right)$ for each compact $K \subset X$. Since $\left.\mathcal{L}\right|_{K}$ is a trivial bundle, $\phi_{K}=M_{a, b}$ for some $a, b \in A_{K}$ and we may suppose $\|a(t)\|=\|b(t)\|$ holds for all $t \in K$. It follows from positivity of $\phi_{t}$ that $b(t)=$ $a(t)^{*}$ (for $t \in K$ ). By the proof of Theorem 6.9 $\phi$ is in the norm closure of $\operatorname{TM}_{c p}(A) \cap \mathrm{TM}_{0}(A)$.

Now suppose that $X=\Delta$. Then by Remark $6.15 \check{H}^{2}(K ; \mathbb{Z})=0$ for all compact subsets $K$ of $\Delta$. By Corollary 6.10 (and Remark 5.10 ) we conclude that $\overline{\overline{\operatorname{TM}_{0}(A)}}=$ $\operatorname{IB}_{0,1}(A)$.

Theorem 6.18. Let $A=\Gamma_{0}(\mathcal{E})$ be an $n$-homogeneous $C^{*}$-algebra with $n \geq 2$.

(a) If $X$ is second-countable with $\operatorname{dim} X<2$ or if $X$ is (homeomorphic to) a subset of a non-compact connected 2-manifold, then both $\operatorname{TM}_{0}(A)$ and $\operatorname{TM}(A)$ are norm closed.

(b) If there is a nonempty open subset of $X$ homeomorphic to (an open subset of) $\mathbb{R}^{d}$ for some $d \geq 3$, then $\operatorname{TM}_{0}(A)$ and $\operatorname{TM}(A)$ both fail to be norm closed.

Proof. (a) follows from Corollary 5.16 (a) and Proposition 6.1.

For (b) we first choose an open subset $U \subset X$ for which $\left.\mathcal{E}\right|_{U}$ is trivial and such that $U$ can be considered as an open set in $\mathbb{R}^{d}(d \geq 3)$. Choose any open subset $V$ of $U$ that has the homotopy type of the set $\Omega$ of Proposition 6.16. In particular, $V$ is of type $K(\mathbb{Q}, 1)$, so it allows a phantom complex line bundle (Proposition 6.14). Now apply Corollary 6.12

Remark 6.19. Suppose that $A=\Gamma_{0}(\mathcal{E})$ is a separable $n$-homogeneous $C^{*}$-algebra with $n \geq 2$ such that $\operatorname{dim} X=d<\infty$. By Remark 5.18 (applied to an $\mathbb{M}_{n^{-}}$ bundle $\mathcal{E}$ ) $A$ has the finite type property. Hence, by [25, Theorem 1.1], we have $\operatorname{IB}(A)=\mathcal{E} \ell(A)$. If $X$ is either a $\mathrm{CW}$-complex or a subset of a $d$-manifold, the following relations between $\operatorname{TM}_{0}(A), \overline{\overline{\operatorname{TM}_{0}(A)}}$ and $\operatorname{IB}_{0,1}(A)$ occur:

(a) If $d<2$ we always have $\operatorname{TM}_{0}(A)=\overline{\overline{\mathrm{TM}_{0}(A)}}=\mathrm{IB}_{0,1}(A)$ (Corollary 5.16 (a)).

(b) If $d=2$ we have four possibilities:

(i) $\mathrm{TM}_{0}(A)=\overline{\overline{\operatorname{TM}_{0}(A)}}=\operatorname{IB}_{0,1}(A)$. This happens e.g. whenever $X$ is a subset of a non-compact connected 2-manifold (Corollary 5.16 (a)).

(ii) $\operatorname{TM}_{0}(A)=\overline{\overline{\operatorname{TM}_{0}(A)}} \subsetneq \operatorname{IB}_{0,1}(A)$. This happens e.g. for $A=C\left(X, \mathbb{M}_{n}\right)$, where $X=\mathbb{S}^{2}$ (by Example 5.14 and since any proper open subset $U$ of $\mathbb{S}^{2}$ is homeomorphic to an open subset of $\mathbb{R}^{2}$, so $\check{H}^{2}(U ; \mathbb{Z})=0$ by Proposition 5.20).

(iii) $\operatorname{TM}_{0}(A) \subsetneq \overline{\overline{\operatorname{TM}_{0}(A)}}=\operatorname{IB}_{0,1}(A)$. This happens e.g. for $A=C_{0}\left(X, \mathbb{M}_{n}\right)$, where $X=\Delta$ is the standard model of $K(\mathbb{Q}, 1)$ (Proposition 6.17).

(iv) $\operatorname{TM}_{0}(A) \subsetneq \overline{\overline{\operatorname{TM}_{0}(A)}} \subsetneq \operatorname{IB}_{0,1}(A)$. This happens e.g. for $A=C_{0}\left(X, \mathbb{M}_{n}\right)$, where $X$ is the topological disjoint union $\mathbb{S}^{2} \sqcup \Delta$ (by Proposition 6.17 Corollary 6.10 and Example 5.14).

(c) If $d>2$ we always have $\operatorname{TM}_{0}(A) \subsetneq \overline{\overline{\mathrm{TM}_{0}(A)}} \subsetneq \mathrm{IB}_{0,1}(A)$ (by Theorem 6.18

(b) and the fact that $X$ must contain an open subset homeomorphic to $\mathbb{R}^{d}$

- if $X$ is a subset of a $d$-manifold, this follows from [9, Theorems 1.7.7, 1.8.9 and 4.1.9]). 
Similar relations occur between $\operatorname{TM}(A), \overline{\overline{\mathrm{TM}(A)}}$ and $\mathrm{IB}_{1}(A)$ in parts (a) and (c) of the above cases.

Acknowledgement: We are very grateful to Mladen Bestvina for his extensive and generous help with many of the topological aspects of the paper.

\section{REFERENCES}

[1] C. A. Akemann, G. K. Pedersen and J. Tomiyama, Multipliers of $C^{*}$-algebras, J. Functional Analysis 13 (1973) 277-301.

[2] P. Ara and M. Mathieu, Local multipliers of $C^{*}$-algebras, Springer Monographs in Mathematics, Springer-Verlag London, Ltd., London (2003), URL http://dx.doi.org/10.1007/978-1-4471-0045-4

[3] R. J. Archbold and D. W. B. Somerset, Multiplier algebras of $C_{0}(X)$-algebras, Münster J. Math. 4 (2011) 73-100.

[4] -, Separation properties in the primitive ideal space of a multiplier algebra, Israel J. Math. 200 (2014) 389-418, URL http://dx.doi.org/10.1007/s11856-014-0022-6

[5] A. Chatterjee and R. R. Smith, The central Haagerup tensor product and maps between von Neumann algebras, J. Funct. Anal. 112 (1993) 97-120, URL http://dx.doi.org/10.1006/jfan.1993.1027

[6] G. R. Conner, M. H. Meilstrup and D. Repovš, The Geometry and Fundamental Groups of Solenoid Complements (2012), arXiv:1212.0128

[7] O. Cornea, G. Lupton, J. Oprea and D. Tanré, Lusternik-Schnirelmann category, Mathematical Surveys and Monographs, vol. 103, American Mathematical Society, Providence, RI (2003), URL http://dx.doi.org/10.1090/surv/103

[8] M. J. Dupré, Classifying Hilbert bundles, J. Functional Analysis 15 (1974) 244-278.

[9] R. Engelking, Dimension theory, North-Holland Publishing Co., Amsterdam-Oxford-New York; PWN-Polish Scientific Publishers, Warsaw (1978), translated from the Polish and revised by the author; North-Holland Mathematical Library, 19.

[10] J. M. G. Fell, The structure of algebras of operator fields, Acta Math. 106 (1961) 233-280.

[11] J. M. G. Fell and R. S. Doran, Representations of *-algebras, locally compact groups, and Banach *-algebraic bundles. Vol. 1, Pure and Applied Mathematics, vol. 125, Academic Press, Inc., Boston, MA (1988), basic representation theory of groups and algebras.

[12] I. Gogić, Derivations which are inner as completely bounded maps, Oper. Matrices 4 (2010) 193-211, URL http://dx.doi.org/10.7153/oam-04-09

[13] - Elementary operators and subhomogeneous $C^{*}$-algebras, Proc. Edinb. Math. Soc. (2) 54 (2011) 99-111, URL http://dx.doi.org/10.1017/S0013091509001114.

[14] - Elementary operators and subhomogeneous $C^{*}$-algebras II, Banach J. Math. Anal. 5 (2011) 181-192, URL http://dx.doi.org/10.15352/bjma/1313362989.

[15] -, On derivations and elementary operators on $C^{*}$-algebras, Proc. Edinb. Math. Soc. (2) 56 (2013) 515-534, URL http://dx.doi.org/10.1017/S0013091512000302

[16] A. Hatcher, Algebraic topology, Cambridge University Press, Cambridge (2002).

[17] S.-t. Hu, Theory of retracts, Wayne State University Press, Detroit (1965).

[18] D. Husemoller, Fibre bundles, Graduate Texts in Mathematics, vol. 20, Springer-Verlag, New York, third ed. (1994), URL http://dx.doi.org/10.1007/978-1-4757-2261-1

[19] R. V. Kadison, E. C. Lance and J. R. Ringrose, Derivations and automorphisms of operator algebras. II, J. Functional Analysis 1 (1967) 204-221.

[20] I. Kaplansky, The structure of certain operator algebras, Trans. Amer. Math. Soc. 70 (1951) 219-255.

[21] D. W. Kribs, A quantum computing primer for operator theorists, Linear Algebra Appl. 400 (2005) 147-167, URL http://dx.doi.org/10.1016/j.laa.2004.11.010

[22] E. C. Lance, Automorphisms of certain operator algebras, Amer. J. Math. 91 (1969) 160-174.

[23] B. Magajna, A transitivity theorem for algebras of elementary operators, Proc. Amer. Math. Soc. 118 (1993) 119-127, URL http://dx.doi.org/10.2307/2160018

[24] - , Pointwise approximation by elementary complete contractions, Proc. Amer. Math. Soc. 137 (2009) 2375-2385, URL http://dx.doi.org/10.1090/S0002-9939-09-09781-0

[25] —, Uniform approximation by elementary operators, Proc. Edinb. Math. Soc. (2) 52 (2009) 731-749, URL http://dx.doi.org/10.1017/S0013091507001290 
[26] -, Approximation of maps on $C^{*}$-algebras by completely contractive elementary operators, in Elementary operators and their applications, Oper. Theory Adv. Appl., vol. 212, Birkhäuser/Springer Basel AG, Basel (2011) pp. 25-39, URL http://dx.doi.org/10.1007/978-3-0348-0037-2_3

[27] C. A. McGibbon, Phantom maps, in Handbook of algebraic topology, North-Holland, Amsterdam (1995) pp. 1209-1257, URL http://dx.doi.org/10.1016/B978-044481779-2/50026-2

[28] T. Napier and M. Ramachandran, Elementary construction of exhausting subsolutions of elliptic operators, Enseign. Math. (2) 50 (2004) 367-390.

[29] V. Paulsen, Completely bounded maps and operator algebras, Cambridge Studies in Advanced Mathematics, vol. 78, Cambridge University Press, Cambridge (2002).

[30] J. Phillips and I. Raeburn, Automorphisms of $C^{*}$-algebras and second Čech cohomology, Indiana Univ. Math. J. 29 (1980) 799-822, URL http://dx.doi.org/10.1512/iumj.1980.29.29058.

[31] J. Phillips, I. Raeburn and J. L. Taylor, Automorphisms of certain $C^{*}$-algebras and torsion in second Čech cohomology, Bull. London Math. Soc. 14 (1982) 33-38, URL http://dx.doi.org/10.1112/blms/14.1.33

[32] N. C. Phillips, Recursive subhomogeneous algebras, Trans. Amer. Math. Soc. 359 (2007) 4595-4623 (electronic), URL http://dx.doi.org/10.1090/S0002-9947-07-03850-0

[33] I. Raeburn and D. P. Williams, Morita equivalence and continuous-trace $C^{*}$-algebras, Mathematical Surveys and Monographs, vol. 60, American Mathematical Society, Providence, RI (1998), URL http://dx.doi.org/10.1090/surv/060

[34] M.-s. B. Smith, On automorphism groups of $C^{*}$-algebras, Trans. Amer. Math. Soc. 152 (1970) 623-648.

[35] D. W. B. Somerset, The inner derivations and the primitive ideal space of a $C^{*}$-algebra, J. Operator Theory 29 (1993) 307-321.

[36] R. M. Timoney, Computing the norms of elementary operators, Illinois J. Math. 47 (2003) 1207-1226, URL http://projecteuclid.org/euclid.ijm/1258138100

[37] J. Tomiyama and M. Takesaki, Applications of fibre bundles to the certain class of $C^{*}$ algebras, Tôhoku Math. J. (2) 13 (1961) 498-522.

[38] J. Wiegold, $\operatorname{Ext}(Q, Z)$ is the additive group of real numbers, Bull. Austral. Math. Soc. 1 (1969) 341-343.

(1) School of Mathematics, Trinity College, Dublin 2, Ireland

(2) Department of Mathematics, University of Zagreb, BijeničKa cesta 30, Zagreb, 10000, Croatia

E-mail address: ilja@maths.tcd.ie, ilja@math.hr

E-mail address: richardt@maths.tcd.ie 